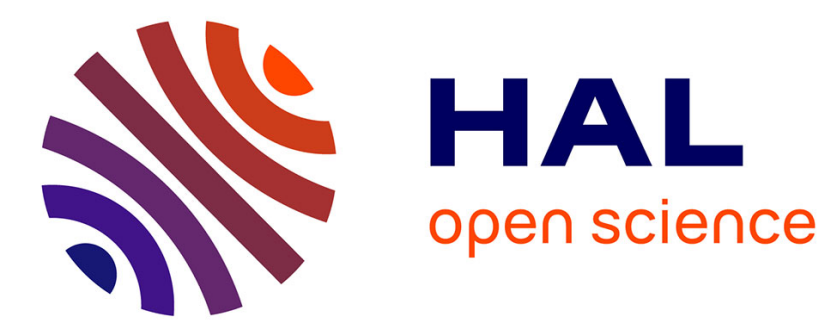

\title{
TVaR-based capital allocation with copulas
}

Mathieu Bargès, Hélène Cossette, Etienne Marceau

\section{To cite this version:}

Mathieu Bargès, Hélène Cossette, Etienne Marceau. TVaR-based capital allocation with copulas. 2009. hal-00431265

\section{HAL Id: hal-00431265 https://hal.science/hal-00431265}

Preprint submitted on 11 Nov 2009

HAL is a multi-disciplinary open access archive for the deposit and dissemination of scientific research documents, whether they are published or not. The documents may come from teaching and research institutions in France or abroad, or from public or private research centers.
L'archive ouverte pluridisciplinaire HAL, est destinée au dépôt et à la diffusion de documents scientifiques de niveau recherche, publiés ou non, émanant des établissements d'enseignement et de recherche français ou étrangers, des laboratoires publics ou privés. 


\title{
TVaR-based capital allocation with copulas
}

\author{
Mathieu Bargès* $\quad$ Hélène Cossette ${ }^{\ddagger} \quad$ Étienne Marceau ${ }^{\ddagger}$
}

\begin{abstract}
Because of regulation projects from control organizations such as the European solvency II reform and recent economic events, insurance companies need to consolidate their capital reserve with coherent amounts allocated to the whole company and to each line of business. The present study considers an insurance portfolio consisting of several lines of risk which are linked by a copula and aims to evaluate not only the capital allocation for the overall portfolio but also the contribution of each risk over their aggregation. We use the tail value at risk (TVaR) as risk measure. The handy form of the FGM copula permits an exact expression for the TVaR of the sum of the risks and for the TVaR-based allocations when claim amounts are exponentially distributed and distributed as a mixture of exponentials. We first examine the bivariate model and then the multivariate case. We also show how to approximate the TVaR of the aggregate risk and the contribution of each risk when using any copula.
\end{abstract}

Keywords: Capital allocation; Tail value at risk; Dependence models; Copulas; Discretization methods

\section{Introduction}

In recent years, a lot of research has focused on insurance capital allocation. Indeed the European Solvency II project and the recent events encourage insurance companies to consolidate their financial reserves and investments. Risk measures are well-known tools to determine the capital amount that has to be allocated to a risk portfolio. Artzner et al. (1999) proposed an axiomal definition of a coherent risk measure that can be used for allocation issues. This coherence property has also been discussed in Wang (2002). Using their definition, Artzner et al. (1999) proposed the tail value at risk (TVaR), also called expected shortfall (ES), as a coherent alternative to the non-coherent risk measure value at risk (VaR). Applied to continuous random variables, the TVaR can identically be defined as the conditional tail expectation (CTE). But these two risk measures differ in discrete contexts where the CTE is no longer coherent. The differences between these definitions and properties have been highlighted in Acerbi et al. (2001) and Acerbi and Tasche (2002).

In the literature on capital allocation, continuous situations are widely studied in contrast with discrete cases. That is why most of the references speak in terms of CTE. The capital

\footnotetext{
* Université de Lyon, Université Lyon 1, ISFA, laboratoire SAF

${ }^{\ddagger}$ École d'Actuariat, Université Laval, Québec, Canada
} 
allocation principle has first been introduced by Tasche (1999) where the capital allocated to each risk is expressed in terms of the CTE of the aggregate risk. This top down allocation method has then been used to provide several closed formulae and approximations of the CTE and the CTE-based allocations for different types of multivariate continuous distributions. The first multivariate top down model was considered by Panjer (2002) where the risks have a multivariate normal distribution. This work has been extended to a multivariate elliptical distribution in Landsman and Valdez (2003) and in Dhaene et al. (2008). A multivariate gamma distribution for risks has been studied in Furman and Landsman (2005) as well as a multivariate Tweedie distribution in Furman and Landsman (2007). In these papers, explicit expressions for the CTE and the CTE-based allocation are derived. Other closed form expressions for the CTE of the sum of multivariate phase-type distributed risks and the contribution of one risk to the portfolio have been given in Cai and Li (2005). More recently, Chiragiev and Landsman (2007) found a CTE and CTE-based allocation for multivariate Pareto risks. Further information on the CTE-based allocation of risk capital can be found in Kim (2007).

In most papers mentioned above, the dependence between the different lines of business of the insurance company is due to the construction of a multivariate distribution. In the present paper, we propose introducing dependence with a copula. Copulas are currently seen as effective and flexible tools to represent dependence between random variables. Furthermore, in order to have the risk measure coherence property in every continuous and discrete situation, we propose using the TVaR as defined in Acerbi et al. (2001) and Acerbi and Tasche (2002) to develop a top down approach of the capital allocation. Indeed, the Committee of European Insurance and Occupational Pensions Supervisors (CEIOPS) advises in the Solvency II context the use of the TVaR for the evaluation of the Solvency Capital Requirement (SCR); see CEIOPS (2006) and CEIOPS (2007).

First, closed form expressions for the TVaR and then the TVaR-based contribution of one risk over the aggregation of all risks are obtained when the Farlie-Gumbel-Morgenstern copula describes the dependence between the risk marginals. With most copulas introducing dependence between different risks however, we are not able to reach closed form expressions. Consequently, we also present approximation methods to evaluate the TVaR and the TVaR-based allocation by the use of different discretization methods of continuous random variables which are applicable with any copula and any marginals.

In the first section, we give the general definitions for the tail value at risk of the aggregate risk and the contribution of one of the risks. The second section deals with the application of the TVaR-based allocation rule using the FGM copula and exponential distributed risks. We first consider two lines of business and then pursue to a multivariate context. We widen our results to risks that are distributed as mixture of exponentials in section 3. For these two last sections, we are able to have closed form expressions for both the TVaR and the individual risk contribution based on it. Then, we expose approximation methods for the TVaR and TVaR-based allocation when the dependence structure is defined by any copula. The results are illustrated with numerical applications. 


\section{Definition of the TVaR and the TVaR-based allocation}

In this section, we define the tail value at risk (TVaR) for the aggregate risk and the TVaR-based allocation rule. We consider the aggregate claim amount (or loss) $S$ of a portfolio of $n$ risks. The claim amount (or loss) for risk $i$ is denoted by $X_{i}$. Thus we have $S=X_{1}+\ldots+X_{n}$ where all $X_{i}$ 's are non-negative random variables.

The value at risk at level $\kappa, 0<\kappa<1$, of $\mathrm{S}$ is defined by

$$
\operatorname{VaR}_{\kappa}(S)=\inf \left(x \in \mathbb{R}, F_{S}(x) \geq \kappa\right) .
$$

It is well known that the VaR is a risk measure that is not coherent. Thus we choose to work with

the tail value at risk of $S$ as introduced in Acerbi and Tasche (2002), Schmock and Straumann (1999) and Schmock (2006) at level $\kappa$, for $\kappa \in(0,1)$. Its definition is

$$
\begin{aligned}
\operatorname{TVaR}_{\kappa}(S) & =\frac{1}{1-\kappa} \int_{\kappa}^{1} \operatorname{VaR}_{u}(S) d u \\
& =\frac{\left.E\left[S 1_{\{S>\operatorname{VaR}}(S)\right\}\right]+\operatorname{VaR}_{\kappa}(S)\left(\operatorname{Pr}\left(S \leq \operatorname{VaR} R_{\kappa}(S)\right)-\kappa\right)}{1-\kappa},
\end{aligned}
$$

which is a coherent risk measure. When $S$ is continuous, $\operatorname{Pr}\left(S \leq \operatorname{VaR} R_{\kappa}(S)\right)=\kappa$ which implies that the TVaR is exactly the conditional tail expectation (CTE) meaning $T V a R_{\kappa}(S)=E[S \mid S>$ $\left.\operatorname{VaR}_{\kappa}(S)\right]=C T E(S)$. In financial risk management, the TVaR is called the expected shortfall.

The additivity of the expectation allows the decomposition of the TVaR (CTE) into the sum of TVaR contributions as follows

$$
T \operatorname{Va} R_{\kappa}(S)=\sum_{i=1}^{n} T \operatorname{Va} R_{\kappa}\left(X_{i} ; S\right)
$$

where the TVaR contribution of the $i$ th risk to the total risk represents the part of the capital that is allocated to risk $i$. For $\kappa \in(0,1)$, it can be expressed as

$$
T \operatorname{VaR} R_{\kappa}\left(X_{i} ; S\right)=\frac{E\left[X_{i} \times 1_{\left\{S>V a R_{\kappa}(S)\right\}}\right]+\beta_{S} E\left[X_{i} \times 1_{\left\{S=V a R_{\kappa}(S)\right\}}\right]}{1-\kappa},
$$

with

$$
\beta_{S}= \begin{cases}\frac{\operatorname{Pr}\left(S \leq \operatorname{VaR} R_{\kappa}(S)\right)-\kappa}{\operatorname{Pr}\left(S=\operatorname{VaR} R_{\kappa}(S)\right)}, & \text { if } \operatorname{Pr}\left(S=\operatorname{VaR} R_{\kappa}(S)\right)>0 \\ 0, \text { otherwise. } & \end{cases}
$$


For continuous distributions, we have

$$
\begin{aligned}
\operatorname{TVaR}_{\kappa}\left(X_{i} ; S\right) & =\frac{1}{1-\kappa} E\left[X_{i} \times 1_{\{S>\operatorname{VaR}(S)\}}\right] \\
& =E\left[X_{i} \mid S>\operatorname{VaR} R_{\kappa}(S)\right] \\
& =C T E_{\kappa}\left(X_{i} \mid S\right) .
\end{aligned}
$$

That means that the TVaR-based contribution of one risk is equal to the CTE-based contribution of the same risk when all the marginals are continuous.

Note that

$$
\begin{aligned}
E\left[X_{i} \mid S=s\right] & =E\left[X_{i} \mid X_{1}+\ldots+X_{n}=s\right] \\
& =\int_{0}^{s} x f_{X_{i} \mid S}(x \mid S=s) d x \\
& =\int_{0}^{s} x \frac{f_{X_{i}, S}(x, s)}{f_{S}(s)} d x .
\end{aligned}
$$

Then, the CTE-based contribution can be expressed as

$$
\begin{aligned}
C T E_{\kappa}\left(X_{i} \mid S\right) & =\int_{\operatorname{VaR}_{\kappa}(S)}^{\infty} E\left[X_{i} \mid S=s\right] f_{S \mid S>\operatorname{VaR} R_{\kappa}(S)}(s) d s \\
& =\frac{1}{\operatorname{Pr}(S>\operatorname{VaR}(S))} \int_{\operatorname{VaR}_{\kappa}(S)}^{\infty} E\left[X_{i} \mid S=s\right] f_{S}(s) d s \\
& =\frac{1}{1-\kappa} \int_{\operatorname{VaR}_{\kappa}(S)}^{\infty} \int_{0}^{s} x f_{X_{i}, S}(x, s) d x d s .
\end{aligned}
$$

\section{TVaR and the TVaR-based allocation with exponential margi- nals and the FGM copula}

In this section, we derive the expression for the TVaR and the TVaR-based allocation for two exponentially distributed risks joined by a FGM copula. We also extend the results obtained with this bivariate model to a multivariate model. The exponential distribution is a classical distribution for the risk random variables. Its convenient and practical mathematic properties permit to develop explicit results. We are aware that the FGM copula introduces only light dependence. However, it admits positive as well as negative dependence between a set of random variables. As said in Yeo and Valdez (2006) where the FGM copula is used to link claim variables in a credibility model, even if it can model only weak dependence, the FGM copula permits to assign a unique dependence parameter for each pair or group of risks and allows a more complex dependence structure than most of the copulas which use only one or few parameters. Furthermore, its handy form allows explicit calculus and thus exact results. This copula was also used to describe different correlation relations on the financial markets in Gatfaoui (2005) and Gatfaoui (2007). 


\subsection{The bivariate case}

Let $X_{1}$ and $X_{2}$ be two exponentially distributed random variables representing the claim amounts of two insurance risks. Their cumulative distribution functions (cdf) and probability density functions (pdf) are given by

$$
\begin{aligned}
F_{X_{i}}\left(x_{i}\right) & =1-e^{-\lambda_{i} x_{i}} \\
f_{X_{i}}\left(x_{i}\right) & =\lambda_{i} e^{-\lambda_{i} x_{i}}, \text { for } i=1,2 .
\end{aligned}
$$

In order to simplify our presentation, we restrain our study to the constraints $\lambda_{1} \neq \lambda_{2}, \lambda_{1} \neq$ $2 \lambda_{2}, \lambda_{2} \neq 2 \lambda_{1}$. It is possible to find adjusted results without these constraints by applying a similar method as the one exposed below.

A dependence structure for $\left(X_{1}, X_{2}\right)$ based on the bivariate FGM copula is introduced. The FGM copula is defined by

$$
C_{\theta}^{F G M}\left(u_{1}, u_{2}\right)=u_{1} u_{2}+\theta u_{1} u_{2}\left(1-u_{1}\right)\left(1-u_{2}\right)
$$

for $u_{i} \in[0,1], i=1,2$, and dependence parameter $\theta \in[-1,1]$.

The density of the bivariate FGM copula is

$$
\begin{aligned}
c_{\theta}^{F G M}\left(u_{1}, u_{2}\right) & =\frac{\partial^{2} C_{\theta}^{F G M}\left(u_{1}, u_{2}\right)}{\partial u_{1} \partial u_{2}} \\
& =1+\theta\left(1-2 u_{1}\right)\left(1-2 u_{2}\right) \\
& =1+\theta\left(2 \bar{u}_{1}-1\right)\left(2 \bar{u}_{2}-1\right),
\end{aligned}
$$

where $\bar{u}_{i}=1-u_{i}, i=1,2$.

The TVaR of the aggregate risk $S=X_{1}+X_{2}$ is given in the following proposition.

Proposition 1 Let $X_{1}$ and $X_{2}$ be two exponentially distributed random variables with joint cdf defined by a bivariate FGM copula as follows

$$
F_{X_{1}, X_{2}}\left(x_{1}, x_{2}\right)=C_{\theta}^{F G M}\left(F_{X_{1}}\left(x_{1}\right), F_{X_{2}}\left(x_{2}\right)\right),
$$

with $\theta \in[-1,1]$. Then, the TVaR of the aggregate risk $S=X_{1}+X_{2}$ at level $\kappa, 0<\kappa<1$, is

$$
\begin{aligned}
\operatorname{TVaR}_{\kappa}(S)= & \frac{1}{1-\kappa}\left[(1+\theta) \zeta\left(\operatorname{VaR} R_{\kappa}(S) ; \lambda_{1} ; \lambda_{2}\right)-\theta \zeta\left(\operatorname{VaR}_{\kappa}(S) ; 2 \lambda_{1} ; \lambda_{2}\right)\right. \\
& \left.-\theta \zeta\left(\operatorname{VaR}_{\kappa}(S) ; \lambda_{1} ; 2 \lambda_{2}\right)+\theta \zeta\left(\operatorname{VaR}_{\kappa}(S) ; 2 \lambda_{1} ; 2 \lambda_{2}\right)\right]
\end{aligned}
$$

where $\zeta\left(x ; \gamma_{1}, \gamma_{2}\right)=\frac{\gamma_{2}}{\gamma_{2}-\gamma_{1}} e^{-\gamma_{1} x}\left(x+\frac{1}{\gamma_{1}}\right)+\frac{\gamma_{1}}{\gamma_{1}-\gamma_{2}} e^{-\gamma_{2} x}\left(x+\frac{1}{\gamma_{2}}\right)$ 
Proof. The joint pdf of $\left(X_{1}, X_{2}\right)$ is given by

$$
\begin{aligned}
f_{X_{1}, X_{2}}\left(x_{1}, x_{2}\right)= & c_{\theta}^{F G M}\left(F_{X_{1}}\left(x_{1}\right), F_{X_{2}}\left(x_{2}\right)\right) f_{X_{1}}\left(x_{1}\right) f_{X_{2}}\left(x_{2}\right) \\
= & f_{X_{1}}\left(x_{1}\right) f_{X_{2}}\left(x_{2}\right)+\theta f_{X_{1}}\left(x_{1}\right) f_{X_{2}}\left(x_{2}\right)\left(1-2 F_{X_{1}}\left(x_{1}\right)\right)\left(1-2 F_{X_{2}}\left(x_{2}\right)\right) \\
= & (1+\theta) \lambda_{1} e^{-\lambda_{1} x_{1}} \lambda_{2} e^{-\lambda_{2} x_{2}}-\theta 2 \lambda_{1} e^{-2 \lambda_{1} x_{1}} \lambda_{2} e^{-\lambda_{2} x_{2}} \\
& -\theta \lambda_{1} e^{-\lambda_{1} x_{1}} 2 \lambda_{2} e^{-2 \lambda_{2} x_{2}}+\theta 2 \lambda_{1} e^{-2 \lambda_{1} x_{1}} 2 \lambda_{2} e^{-2 \lambda_{2} x_{2}} .
\end{aligned}
$$

Let $h\left(x, \lambda_{1}, \lambda_{2}\right)$ be the distribution function of a generalized Erlang random variable $X$

$$
h\left(x, \lambda_{1}, \lambda_{2}\right)=\frac{\lambda_{1} \lambda_{2}}{\lambda_{2}-\lambda_{1}} e^{-\lambda_{1} x}+\frac{\lambda_{1} \lambda_{2}}{\lambda_{1}-\lambda_{2}} e^{-\lambda_{2} x} .
$$

Then, the pdf of $S$ can be expressed as a combination of generalized Erlang pdf's

$$
\begin{aligned}
f_{S}(s) & =\int_{0}^{s} f_{X_{1}, S}(x, s) d x \\
& =\int_{0}^{s} f_{X_{1}, X_{2}}(x, s-x) d x \\
& =(1+\theta) h\left(s ; \lambda_{1} ; \lambda_{2}\right)-\theta h\left(s ; 2 \lambda_{1} ; \lambda_{2}\right)-\theta h\left(s ; \lambda_{1} ; 2 \lambda_{2}\right)+\theta h\left(s ; 2 \lambda_{1} ; 2 \lambda_{2}\right),
\end{aligned}
$$

where the first component of $f_{S}(s)$ is

$$
\begin{aligned}
\int_{0}^{s}(1+\theta) \lambda_{1} e^{-\lambda_{1} x} \lambda_{2} e^{-\lambda_{2}(s-x)} d x & =(1+\theta) \lambda_{1} \lambda_{2} e^{-\lambda_{2} s} \int_{0}^{s} e^{x\left(\lambda_{2}-\lambda_{1}\right)} d x \\
& =(1+\theta) \frac{\lambda_{1} \lambda_{2}}{\lambda_{2}-\lambda_{1}} e^{-\lambda_{1} s}+(1+\theta) \frac{\lambda_{1} \lambda_{2}}{\lambda_{1}-\lambda_{2}} e^{-\lambda_{2} s} \\
& =(1+\theta) h\left(s ; \lambda_{1} ; \lambda_{2}\right) .
\end{aligned}
$$

The three other components can be found similarly.

Thus, the TVaR of $S$ takes the form

$$
\begin{aligned}
\operatorname{TVaR}_{\kappa}(S)= & E\left[S \mid S>\operatorname{VaR}_{\kappa}(S)\right] \\
= & \int_{V a R_{\kappa}}^{\infty} s \frac{f_{S}(s)}{\operatorname{Pr}(S>\operatorname{VaR}(S))} d s \\
= & \frac{1}{1-F_{S}\left(\operatorname{VaR}_{\kappa}(S)\right)} \int_{\operatorname{VaR}_{\kappa}(S)}^{\infty} s f_{S}(s) d s \\
= & \frac{1}{1-\kappa} \int_{V^{\prime} R_{\kappa}(S)}^{\infty} s\left[(1+\theta) h\left(s ; \lambda_{1} ; \lambda_{2}\right)-\theta h\left(s ; 2 \lambda_{1} ; \lambda_{2}\right)\right. \\
& \left.-\theta h\left(s ; \lambda_{1} ; 2 \lambda_{2}\right)+\theta h\left(s ; 2 \lambda_{1} ; 2 \lambda_{2}\right)\right] d s .
\end{aligned}
$$


Define

$$
\begin{aligned}
\int_{V a R_{\kappa}(S)}^{\infty} s h\left(s ; \lambda_{1} ; \lambda_{2}\right) d s= & \int_{V a R_{\kappa}(S)}^{\infty} s\left(\frac{\lambda_{1} \lambda_{2}}{\lambda_{2}-\lambda_{1}} e^{-\lambda_{1} s}+\frac{\lambda_{1} \lambda_{2}}{\lambda_{1}-\lambda_{2}} e^{-\lambda_{2} s}\right) d s \\
= & \frac{\lambda_{1} \lambda_{2}}{\lambda_{2}-\lambda_{1}}\left\{\left[-s \frac{e^{-\lambda_{1} s}}{\lambda_{1}}\right]_{V a R_{\kappa}(S)}^{\infty}+\int_{V a R_{\kappa}(S)}^{\infty} \frac{e^{-\lambda_{1} s}}{\lambda_{1}} d s\right\} \\
& +\frac{\lambda_{1} \lambda_{2}}{\lambda_{1}-\lambda_{2}}\left\{\left[-s \frac{e^{-\lambda_{2} s}}{\lambda_{2}}\right]_{V a R_{\kappa}(S)}^{\infty} \int_{V a R_{\kappa}(S)}^{\infty} \frac{e^{-\lambda_{2} s}}{\lambda_{2}} d s\right\} \\
= & \frac{\lambda_{1} \lambda_{2}}{\lambda_{2}-\lambda_{1}}\left(\operatorname{VaR}(S) \frac{e^{-\lambda_{1} V a R_{\kappa}(S)}}{\lambda_{1}}+\frac{e^{-\lambda_{1} V a R_{\kappa}(S)}}{\lambda_{1}^{2}}\right) \\
& +\frac{\lambda_{1} \lambda_{2}}{\lambda_{1}-\lambda_{2}}\left(\operatorname{VaR} R_{\kappa}(S) \frac{e^{-\lambda_{2} V a R_{\kappa}(S)}}{\lambda_{2}}+\frac{e^{-\lambda_{2} V a R_{\kappa}(S)}}{\lambda_{2}^{2}}\right) \\
= & \frac{\lambda_{2}}{\lambda_{2}-\lambda_{1}} e^{-\lambda_{1} \operatorname{VaR} R_{\kappa}(S)}\left(\operatorname{VaR}(S)+\frac{1}{\lambda_{1}}\right)+\frac{\lambda_{1}}{\lambda_{1}-\lambda_{2}} e^{-\lambda_{2} V a R_{\kappa}(S)}\left(\operatorname{VaR}_{\kappa}(S)+\frac{1}{\lambda_{2}}\right) \\
= & \zeta\left(\operatorname{VaR} R_{\kappa}(S) ; \lambda_{1}, \lambda_{2}\right) .
\end{aligned}
$$

Inserting (5) in (4), we obtain

$$
\begin{aligned}
T \operatorname{Va} R_{\kappa}(S)= & \frac{1}{1-\kappa}\left[(1+\theta) \zeta\left(\operatorname{Va} R_{\kappa}(S) ; \lambda_{1} ; \lambda_{2}\right)-\theta \zeta\left(\operatorname{Va} R_{\kappa}(S) ; 2 \lambda_{1} ; \lambda_{2}\right)\right. \\
& \left.-\theta \zeta\left(\operatorname{Va} R_{\kappa}(S) ; \lambda_{1} ; 2 \lambda_{2}\right)+\theta \zeta\left(\operatorname{VaR} R_{\kappa}(S) ; 2 \lambda_{1} ; 2 \lambda_{2}\right)\right]
\end{aligned}
$$

with

$$
\zeta\left(x ; \gamma_{1}, \gamma_{2}\right)=\frac{\gamma_{2}}{\gamma_{2}-\gamma_{1}} e^{-\gamma_{1} x}\left(x+\frac{1}{\gamma_{1}}\right)+\frac{\gamma_{1}}{\gamma_{1}-\gamma_{2}} e^{-\gamma_{2} x}\left(x+\frac{1}{\gamma_{2}}\right)
$$

A closed form expression for the TVaR-based capital attributed to risk $i, i=1,2$, is given in the next proposition.

Proposition 2 Let $X_{1}$ and $X_{2}$ be two exponentially distributed random variables with joint cdf defined by a bivariate FGM copula. Then, the TVaR-based contribution of risk $i, i=1,2$, to the aggregate risk $S=X_{1}+X_{2}$ at level $\kappa, 0<\kappa<1$, is

$$
\begin{aligned}
T V a R_{\kappa}\left(X_{i} ; S\right)= & \frac{1}{1-\kappa}\left[(1+\theta) \xi\left(\operatorname{Va} R_{\kappa}(S) ; \lambda_{i} ; \lambda_{j}\right)-\theta \xi\left(\operatorname{VaR} R_{\kappa}(S) ; 2 \lambda_{i} ; \lambda_{j}\right)\right. \\
& \left.-\theta \xi\left(\operatorname{VaR}_{\kappa}(S) ; \lambda_{i} ; 2 \lambda_{j}\right)+\theta \xi\left(\operatorname{VaR}_{\kappa}(S) ; 2 \lambda_{i} ; 2 \lambda_{j}\right)\right]
\end{aligned}
$$


where $\xi\left(x ; \gamma_{i} ; \gamma_{j}\right)=\frac{\gamma_{j} e^{-\gamma_{i} x}\left(x+\frac{1}{\gamma_{i}}\right)}{\gamma_{j}-\gamma_{i}}-\frac{\gamma_{j} e^{-\gamma_{i} x}-\gamma_{i} e^{-\gamma_{j} x}}{\left(\gamma_{j}-\gamma_{i}\right)^{2}}$ and $i \neq j$.

Proof. Let $i=1$ and $j=2$. Recall that for continuous random variables the TVaR-based allocation is equal to the CTE-based allocation. From (1), we have

$$
\begin{aligned}
\operatorname{TVaR}_{\kappa}\left(X_{1} ; S\right) & =E\left[X_{1} \mid S>\operatorname{VaR}(S)\right] \\
& =\frac{1}{\operatorname{Pr}(S>\operatorname{VaR}(S))} \int_{\operatorname{VaR}_{\kappa}(S)}^{\infty} \int_{0}^{s} x f_{X_{1}, S}(x, s) d x d s
\end{aligned}
$$

where

$$
\begin{aligned}
\int_{0}^{s} x f_{X_{1}, S}(x, s) d x= & \int_{0}^{s} x f_{X_{1}, X_{2}}(x, s-x) d x \\
= & \int_{0}^{s} x\left((1+\theta) \lambda_{1} e^{-\lambda_{1} x} \lambda_{2} e^{-\lambda_{2}(s-x)}-\theta 2 \lambda_{1} e^{-\lambda_{1} x} \lambda_{2} e^{-\lambda_{2}(s-x)}\right. \\
& \left.-\theta \lambda_{1} e^{-\lambda_{1} x} 2 \lambda_{2} e^{-\lambda_{2}(s-x)}+\theta 2 \lambda_{1} e^{-\lambda_{1} x} 2 \lambda_{2} e^{-\lambda_{2}(s-x)}\right) d x \\
= & (1+\theta) \lambda_{1} \lambda_{2}\left(\frac{s e^{-\lambda_{1} s}}{\lambda_{2}-\lambda_{1}}-\frac{e^{-\lambda_{1} s}-e^{-\lambda_{2} s}}{\left(\lambda_{2}-\lambda_{1}\right)^{2}}\right) \\
& -\theta 2 \lambda_{1} \lambda_{2}\left(\frac{s e^{-2 \lambda_{1} s}}{\lambda_{2}-2 \lambda_{1}}-\frac{e^{-2 \lambda_{1} s}-e^{-\lambda_{2} s}}{\left(\lambda_{2}-2 \lambda_{1}\right)^{2}}\right) \\
& -\theta \lambda_{1} 2 \lambda_{2}\left(\frac{s e^{-\lambda_{1} s}}{2 \lambda_{2}-\lambda_{1}}-\frac{e^{-\lambda_{1} s}-e^{-2 \lambda_{2} s}}{\left(2 \lambda_{2}-\lambda_{1}\right)^{2}}\right) \\
& +\theta 2 \lambda_{1} 2 \lambda_{2}\left(\frac{s e^{-2 \lambda_{1} s}}{2 \lambda_{2}-2 \lambda_{1}}-\frac{e^{-2 \lambda_{1} s}-e^{-2 \lambda_{2} s}}{\left(2 \lambda_{2}-2 \lambda_{1}\right)^{2}}\right)
\end{aligned}
$$

The first component on the right-hand side of the last equality is given by

$$
\begin{aligned}
\int_{0}^{s} x(1+\theta) \lambda_{1} e^{-\lambda_{1} x} \lambda_{2} e^{-\lambda_{2}(s-x)} d x & =(1+\theta) \lambda_{1} \lambda_{2} e^{-\lambda_{2} s} \int_{0}^{s} x e^{x\left(\lambda_{2}-\lambda_{1}\right)} d x \\
& =(1+\theta) \lambda_{1} \lambda_{2} e^{-\lambda_{2} s}\left(\left[x \frac{e^{x\left(\lambda_{2}-\lambda_{1}\right)}}{\lambda_{2}-\lambda_{1}}\right]_{0}^{s}-\int_{0}^{s} \frac{e^{x\left(\lambda_{2}-\lambda_{1}\right)}}{\lambda_{2}-\lambda_{1}} d x\right) \\
& =(1+\theta) \lambda_{1} \lambda_{2} e^{-\lambda_{2} s}\left(s \frac{e^{s\left(\lambda_{2}-\lambda_{1}\right)}}{\lambda_{2}-\lambda_{1}}-\left[\frac{e^{x\left(\lambda_{2}-\lambda_{1}\right)}}{\left(\lambda_{2}-\lambda_{1}\right)^{2}}\right]_{0}^{s}\right) \\
& =(1+\theta) \lambda_{1} \lambda_{2} e^{-\lambda_{2} s}\left(s \frac{e^{s \lambda_{2}} e^{-s \lambda_{1}}}{\lambda_{2}-\lambda_{1}}-\frac{e^{s \lambda_{2}} e^{-s \lambda_{1}}-1}{\left(\lambda_{2}-\lambda_{1}\right)^{2}}\right) \\
& =(1+\theta) \lambda_{1} \lambda_{2}\left(\frac{s e^{-\lambda_{1} s}}{\lambda_{2}-\lambda_{1}}-\frac{e^{-\lambda_{1} s}-e^{-\lambda_{2} s}}{\left(\lambda_{2}-\lambda_{1}\right)^{2}}\right)
\end{aligned}
$$


Denoting $V=\operatorname{VaR}_{\kappa}(S)$, we have

$$
\begin{aligned}
\operatorname{TVaR}_{\kappa}\left(X_{1} ; S\right)= & \frac{1}{1-F_{S}(V)} \int_{V}^{\infty}\left\{(1+\theta) \lambda_{1} \lambda_{2}\left(\frac{s e^{-\lambda_{1} s}}{\lambda_{2}-\lambda_{1}}-\frac{e^{-\lambda_{1} s}-e^{-\lambda_{2} s}}{\left(\lambda_{2}-\lambda_{1}\right)^{2}}\right)\right. \\
& -\theta 2 \lambda_{1} \lambda_{2}\left(\frac{s e^{-2 \lambda_{1} s}}{\lambda_{2}-2 \lambda_{1}}-\frac{e^{-2 \lambda_{1} s}-e^{-\lambda_{2} s}}{\left(\lambda_{2}-2 \lambda_{1}\right)^{2}}\right)-\theta \lambda_{1} 2 \lambda_{2}\left(\frac{s e^{-\lambda_{1} s}}{2 \lambda_{2}-\lambda_{1}}-\frac{e^{-\lambda_{1} s}-e^{-2 \lambda_{2} s}}{\left(2 \lambda_{2}-\lambda_{1}\right)^{2}}\right) \\
& \left.+\theta 2 \lambda_{1} 2 \lambda_{2}\left(\frac{s e^{-2 \lambda_{1} s}}{2 \lambda_{2}-2 \lambda_{1}}-\frac{e^{-2 \lambda_{1} s}-e^{-2 \lambda_{2} s}}{\left(2 \lambda_{2}-2 \lambda_{1}\right)^{2}}\right)\right\} d s \\
= & \frac{1}{1-F_{S}(V)}\left[(1+\theta)\left(\frac{\lambda_{2} e^{-\lambda_{1} V}\left(V+\frac{1}{\lambda_{1}}\right)}{\lambda_{2}-\lambda_{1}}-\frac{\lambda_{2} e^{-\lambda_{1} V}-\lambda_{1} e^{-\lambda_{2} V}}{\left(\lambda_{2}-\lambda_{1}\right)^{2}}\right)\right. \\
& -\theta\left(\frac{\lambda_{2} e^{-2 \lambda_{1} V}\left(V+\frac{1}{2 \lambda_{1}}\right)}{\lambda_{2}-2 \lambda_{1}}-\frac{\lambda_{2} e^{-2 \lambda_{1} V}-2 \lambda_{1} e^{-\lambda_{2} V}}{\left(\lambda_{2}-2 \lambda_{1}\right)^{2}}\right) \\
& -\theta\left(\frac{2 \lambda_{2} e^{-\lambda_{1} V}\left(V+\frac{1}{\lambda_{1}}\right)}{2 \lambda_{2}-\lambda_{1}}-\frac{2 \lambda_{2} e^{-\lambda_{1} V}-\lambda_{1} e^{-2 \lambda_{2} V}}{\left(2 \lambda_{2}-\lambda_{1}\right)^{2}}\right) \\
& \left.+\theta\left(\frac{2 \lambda_{2} e^{-2 \lambda_{1} V}\left(V+\frac{1}{2 \lambda_{1}}\right)}{2 \lambda_{2}-2 \lambda_{1}}-\frac{2 \lambda_{2} e^{-2 \lambda_{1} V}-2 \lambda_{1} e^{-2 \lambda_{2} V}}{\left(2 \lambda_{2}-2 \lambda_{1}\right)^{2}}\right)\right]
\end{aligned}
$$

We finally obtain

$$
\begin{aligned}
\operatorname{TVaR}_{\kappa}\left(X_{1} ; S\right)= & \frac{1}{1-\kappa}\left[(1+\theta) \xi\left(\operatorname{Va} R_{\kappa}(S) ; \lambda_{1} ; \lambda_{2}\right)-\theta \xi\left(\operatorname{VaR}_{\kappa}(S) ; 2 \lambda_{1} ; \lambda_{2}\right)\right. \\
& \left.-\theta \xi\left(\operatorname{VaR}_{\kappa}(S) ; \lambda_{1} ; 2 \lambda_{2}\right)+\theta \xi\left(\operatorname{Va} R_{\kappa}(S) ; 2 \lambda_{1} ; 2 \lambda_{2}\right)\right]
\end{aligned}
$$

where

$$
\xi\left(x ; \gamma_{1} ; \gamma_{2}\right)=\frac{\gamma_{2} e^{-\gamma_{1} x}\left(x+\frac{1}{\gamma_{1}}\right)}{\gamma_{2}-\gamma_{1}}-\frac{\gamma_{2} e^{-\gamma_{1} x}-\gamma_{1} e^{-\gamma_{2} x}}{\left(\gamma_{2}-\gamma_{1}\right)^{2}} .
$$

The TVaR-based allocation for the second risk is symmetrically given by

$$
\begin{aligned}
T V a R_{\kappa}\left(X_{2} ; S\right)= & \frac{1}{1-\kappa}\left[(1+\theta) \xi\left(\operatorname{VaR}_{\kappa}(S) ; \lambda_{2} ; \lambda_{1}\right)-\theta \xi\left(\operatorname{VaR}_{\kappa}(S) ; 2 \lambda_{2} ; \lambda_{1}\right)\right. \\
& \left.-\theta \xi\left(\operatorname{VaR}_{\kappa}(S) ; \lambda_{2} ; 2 \lambda_{1}\right)+\theta \xi\left(\operatorname{VaR}_{\kappa}(S) ; 2 \lambda_{2} ; 2 \lambda_{1}\right)\right]
\end{aligned}
$$

where

$$
\xi\left(x ; \gamma_{2} ; \gamma_{1}\right)=\frac{\gamma_{1} e^{-\gamma_{2} x}\left(x+\frac{1}{\gamma_{2}}\right)}{\gamma_{1}-\gamma_{2}}-\frac{\gamma_{1} e^{-\gamma_{2} x}-\gamma_{2} e^{-\gamma_{1} x}}{\left(\gamma_{1}-\gamma_{2}\right)^{2}} .
$$


Remark 3 It can be verified that the TVaR of $S$ is the sum of the risk contributions

$$
T \operatorname{Va} R_{\kappa}(S)=\sum_{i=1}^{2} T \operatorname{Va} R_{\kappa}\left(X_{i} ; S\right)
$$

Explicit expressions for the TVaR and the one risk TVaR-based contribution cannot only be obtained in the bivariate case but even for an undefined number of risks.

\subsection{The multivariate case}

Suppose now that there are $n$ different exponential risks joined by a multivariate FGM copula characterized by

$$
C\left(u_{1}, u_{2}, \ldots, u_{n}\right)=u_{1} u_{2} \ldots u_{n}\left(1+\sum_{k=2}^{n} \sum_{1 \leq j_{1}<\ldots<j_{k} \leq n} \theta_{j_{1} j_{2} \ldots j_{k}} \bar{u}_{j_{1}} \bar{u}_{j_{2}} \ldots \bar{u}_{j_{k}}\right),
$$

where $\bar{u}_{(.)}=1-u_{(.)}$(see Nelsen $(2006)$ p.108). We have here $2^{n}-n-1$ copula parameters to describe the dependence between each pair or group of risks.

Its density can be written as

$$
c\left(u_{1}, u_{2}, \ldots, u_{n}\right)=1+\sum_{k=2}^{n} \sum_{1 \leq j_{1}<\ldots<j_{k} \leq n} \theta_{j_{1} j_{2} \ldots j_{k}}\left(2 \bar{u}_{j_{1}}-1\right)\left(2 \bar{u}_{j_{2}}-1\right) \ldots\left(2 \bar{u}_{j_{k}}-1\right) .
$$

As in the bivariate case, we suppose that the parameters of the $n$ exponential risks satisfy the conditions below

$$
\lambda_{i} \neq \lambda_{j} \text { and } \lambda_{i} \neq 2 \lambda_{j} \text { for } i \neq j .
$$

Then, the following proposition holds.

Proposition 4 Let $S_{n}=X_{1}+X_{2}+\ldots+X_{n}$ be the sum of $n$ dependent exponential random variables with joint cdf defined by a multivariate FGM copula as follows

$$
F_{X_{1}, \ldots, X_{n}}\left(x_{1}, \ldots, x_{n}\right)=C_{\theta}^{F G M}\left(F_{X_{1}}\left(x_{1}\right), \ldots, F_{X_{n}}\left(x_{n}\right)\right)
$$

with $\theta \in[-1,1]$. Then, the TVaR of $S_{n}$ at level $\kappa, 0<\kappa<1$, is

$$
\begin{aligned}
\operatorname{TVaR}_{\kappa}\left(S_{n}\right)= & \frac{1}{1-\kappa} \times\left[1+\sum_{k=2}^{n} \sum_{1 \leq j_{1}<\ldots<j_{k} \leq n} \theta_{j_{1} j_{2} \ldots j_{k}} \times\right. \\
& \left.\left(\sum_{l=0}^{k} \sum_{\left(a_{1}, \ldots, a_{k}\right) \in A_{l, k}}(-1)^{l} \zeta\left(\operatorname{VaR} R_{\kappa}\left(S_{n}\right) ; 2^{i_{1}} \lambda_{1}, \ldots, 2^{i_{k}} \lambda_{k}, \lambda_{i_{k+1}}, \ldots \lambda_{i_{n}}\right)\right)\right],
\end{aligned}
$$


where $\zeta\left(x ; \gamma_{1}, \ldots, \gamma_{n}\right)=\sum_{i=1}^{n}\left(\prod_{j=1, j \neq i}^{n} \frac{\gamma_{j}}{\gamma_{j}-\gamma_{i}}\right) e^{-\gamma_{i} x}\left(x+\frac{1}{\gamma_{i}}\right), i_{k+1}, \ldots, i_{n}$ are the missing indexes of $j_{1}, \ldots, j_{k}$ to complete $1, \ldots, n$ and $A_{l, k}$ are the sets of $k$-tuples composed of $l$ zeros and $(k-l)$ ones, for $l=0,1, \ldots, k$ and $k=2, \ldots, n$.

In fact, the $A_{l, k}$ are defined by $A_{0, k}=\left\{(1,1, \ldots, 1)_{1 \times k}\right\}, A_{1, k}=\left\{(1,1, \ldots, 0)_{1 \times k}, \ldots,(0,1, \ldots, 1)_{1 \times k}\right\}$, $A_{2, k}=\left\{(1,1, \ldots, 0,0)_{1 \times k}, \ldots,(0,0, \ldots, 1)_{1 \times k}\right\}, \ldots, A_{k, k}=\left\{(0,0, \ldots, 0)_{1 \times k}\right\}$.

Proof. The joint pdf of $\left(X_{1}, X_{2}, \ldots, X_{n}\right)$ is

$$
\begin{aligned}
f_{X_{1}, X_{2}, \ldots, X_{n}}\left(x_{1}, x_{2}, \ldots, x_{n}\right)= & c\left(F_{X_{1}}\left(x_{1}\right), F_{X_{2}}\left(x_{2}\right), \ldots, F_{X_{n}}\left(x_{n}\right)\right) f_{X_{1}}\left(x_{1}\right) f_{X_{2}}\left(x_{2}\right) \ldots f_{X_{n}}\left(x_{n}\right) \\
= & f_{X_{1}}\left(x_{1}\right) f_{X_{2}}\left(x_{2}\right) \ldots f_{X_{n}}\left(x_{n}\right) \\
& \times\left[1+\sum_{k=2}^{n} \sum_{1 \leq j_{1}<\ldots<j_{k} \leq n} \theta_{j_{1} j_{2} \ldots j_{k}}\left(1-F_{X_{j_{1}}}\left(x_{j_{1}}\right)\right)\left(1-F_{X_{j_{2}}}\left(x_{j_{2}}\right)\right) \ldots\left(1-F_{X_{j_{k}}}\left(x_{j_{k}}\right)\right)\right] .
\end{aligned}
$$

As the $n$ risk random variables are exponentially distributed with parameters $\lambda_{i}, i=1, \ldots, n$ with constraint (7), we have

$$
\begin{aligned}
f_{X_{1}, X_{2}, \ldots, X_{n}}\left(x_{1}, x_{2}, \ldots, x_{n}\right)=\lambda_{1} e^{-\lambda_{1} x_{1}} \lambda_{2} e^{-\lambda_{2} x_{2}} \ldots \lambda_{n} e^{-\lambda_{n} x_{n}} \\
\quad \times\left[1+\sum_{k=2}^{n} \sum_{1 \leq j_{1}<\ldots<j_{k} \leq n} \theta_{j_{1} j_{2} \ldots j_{k}}\left(2 e^{-\lambda_{j_{1}} x_{j_{1}}}-1\right)\left(2 e^{-\lambda_{j_{2}} x_{j_{2}}}-1\right) \ldots\left(2 e^{-\lambda_{j_{n}} x_{j_{n}}}-1\right)\right] \\
=\nu\left(x_{1}, x_{2}, \ldots, x_{n} ; \lambda_{1}, \lambda_{2}, \ldots, \lambda_{n}\right)+\sum_{k=2}^{n} \sum_{1 \leq j_{1}<\ldots<j_{k} \leq n} \theta_{j_{1} j_{2} \ldots j_{k}} \\
\quad \times\left[\sum_{l=0}^{k} \sum_{\left(a_{1}, \ldots, a_{k}\right) \in A_{l, k}}(-1)^{l} \nu\left(x_{j_{1}}, x_{j_{2}}, \ldots, x_{j_{k}}, x_{i_{k+1}}, \ldots, x_{i_{n}} ; 2^{a_{1}} \lambda_{j_{1}}, 2^{a_{2}} \lambda_{j_{2}}, \ldots, 2^{a_{k}} \lambda_{j_{k}}, \lambda_{i_{k+1}}, \ldots, \lambda_{i_{n}}\right)\right],
\end{aligned}
$$

where $i_{k+1}, \ldots, i_{n}$ and $A_{l, k}$ are defined as in the proposition and

$$
\nu\left(x_{1}, \ldots, x_{n} ; \gamma_{1}, \ldots, \gamma_{n}\right)=\gamma_{1} e^{-\gamma_{1} x_{1}} \times \gamma_{2} e^{-\gamma_{2} x_{2}} \times \ldots \times \gamma_{n} e^{-\gamma_{n} x_{n}} .
$$

Given that

$$
\begin{aligned}
& f_{S_{n}}(s)=\int_{0}^{s} \int_{0}^{s-x_{1}} \ldots \int_{0}^{s-x_{1}-\ldots-x_{n-1}} f_{X_{1}, X_{2}, \ldots, X_{n-1}, X_{n}}\left(x_{1}, x_{2}, \ldots, x_{n-1}, s-x_{1}-x_{2}-\ldots-x_{n-1}\right) d x_{1} d x_{2} \ldots d x_{n-1} \\
& =\int_{0}^{s} \int_{0}^{s-x_{1}} \ldots \int_{0}^{s-x_{1}-\ldots-x_{n-2}} f_{X_{1}, X_{2}, \ldots, X_{n-2}, X_{n-1}+X_{n}}\left(x_{1}, x_{2}, \ldots, x_{n-2}, s-x_{1}-x_{2}-\ldots-x_{n-2}\right) d x_{1} d x_{2} \ldots d x_{n-2} \\
& =\ldots \\
& =\int_{0}^{s} f_{X_{1}, X_{2}+\ldots+X_{n}}\left(x_{1}, s-x_{1}\right) d x_{1}
\end{aligned}
$$


and that

$$
\begin{aligned}
\int_{0}^{s} \int_{0}^{s-x_{1}} \ldots \int_{0}^{s-x_{1}-\ldots-x_{n-1}} \nu\left(x_{1}, x_{2}, \ldots, x_{n} ; \gamma_{1}, \gamma_{2}, \ldots, \gamma_{n}\right) d x_{1} d x_{2} \ldots d x_{n-1} & =\sum_{i=1}^{n}\left(\prod_{j=1, j \neq i}^{n} \frac{\gamma_{j}}{\gamma_{j}-\gamma_{i}}\right) \gamma_{i} e^{-\gamma_{i} s} \\
& =h\left(s ; \gamma_{1}, \gamma_{2}, \ldots \gamma_{n}\right)
\end{aligned}
$$

one can write

$$
\begin{aligned}
f_{S_{n}}(s)= & h\left(s ; \lambda_{1}, \ldots, \lambda_{n}\right) \\
& +\sum_{k=2}^{n} \sum_{1 \leq j_{1}<\ldots<j_{k} \leq n} \theta_{j_{1} j_{2} \ldots j_{k}}\left(\sum_{l=0}^{k} \sum_{\left(a_{1}, \ldots, a_{k}\right) \in A_{l, k}}(-1)^{l} h\left(s ; 2^{a_{1}} \lambda_{j_{1}}, 2^{a_{2}} \lambda_{j_{2}}, \ldots, 2^{a_{k}} \lambda_{j_{k}}, \lambda_{i_{k+1}}, \ldots \lambda_{i_{n}}\right)\right) .
\end{aligned}
$$

Then, the TVaR of $S_{n}$ for $n \geq 2$ and $0<\kappa<1$ is

$$
\begin{aligned}
\operatorname{TVaR}_{\kappa}\left(S_{n}\right)= & E\left[S_{n} \mid S_{n}>\operatorname{VaR} R_{\kappa}\left(S_{n}\right)\right] \\
= & \int_{V_{a} R_{\kappa}}^{\infty} s \frac{f_{S_{n}}(s)}{\operatorname{Pr}\left(S_{n}>\operatorname{VaR}\left(R_{\kappa}\right)\right)} d s \\
= & \frac{1}{1-\kappa} \int_{\operatorname{VaR_{\kappa }}\left(S_{n}\right)}^{\infty} s f_{S_{n}}(s) d s \\
= & \frac{1}{1-\kappa} \times\left[1+\sum_{k=2}^{n} \sum_{1 \leq j_{1}<\ldots<j_{k} \leq n} \theta_{j_{1} j_{2} \ldots j_{k}} \times\right. \\
& \left.\left(\sum_{l=0}^{k} \sum_{\left(a_{1}, \ldots, a_{k}\right) \in A_{l, k}}(-1)^{l} \zeta\left(\operatorname{VaR} R_{\kappa}\left(S_{n}\right) ; 2^{i_{1}} \lambda_{1}, \ldots, 2^{i_{k}} \lambda_{k}, \lambda_{i_{k+1}}, \ldots \lambda_{i_{n}}\right)\right)\right],
\end{aligned}
$$

where

$$
\zeta\left(x ; \gamma_{1}, \ldots, \gamma_{n}\right)=\sum_{i=1}^{n}\left(\prod_{j=1, j \neq i}^{n} \frac{\gamma_{j}}{\gamma_{j}-\gamma_{i}}\right) e^{-\gamma_{i} x}\left(x+\frac{1}{\gamma_{i}}\right) .
$$

As in the bivariate case, the capital allocation for risk $i$ can also be explicitly given. In order to find that expression, we first need to introduce the $n$th order divided difference of a function $f$ as in Chiragiev and Landsman (2007). Consider $x_{1}, x_{2}, \ldots, x_{n}, x_{n+1}$ arbitrary points such that $x_{i} \neq x_{j}$ for $i \neq j$. The $n$th order divided difference of $f$ on $x_{1}, x_{2}, \ldots, x_{n}, x_{n+1}$ is defined by

$$
f\left(x_{1}, x_{2}, \ldots, x_{n}, x_{n+1}\right)=\sum_{i=1}^{n+1} \frac{f\left(x_{i}\right)}{\prod_{j \neq i}\left(x_{i}-x_{j}\right)} .
$$

The following proposition gives the expression of the TVaR-based allocation. 
Proposition 5 Let $X_{1}, \ldots, X_{n}$ be $n$ exponentially distributed random variables with joint cdf defined by a multivariate FGM copula. Then, the TVaR-based contribution of risk $i, i=1, \ldots, n$, to the sum $S_{n}=X_{1}+\ldots+X_{n}$ at level $\kappa, 0<\kappa<1$, is

$$
\begin{aligned}
\operatorname{TVaR}_{\kappa}\left(X_{i} ; S_{n}\right)= & \frac{(-1)^{n-1} \Lambda}{1-\kappa}\left[\bar{H}_{i}\left(\operatorname{VaR}_{\kappa}\left(S_{n}\right) ; \lambda_{1} ; \ldots ; \lambda_{n}\right)+\sum_{k=2}^{n} \sum_{1 \leq j_{1}<\ldots<j_{k} \leq n \bigcap i \in\left\{j_{1}, \ldots, j_{k}\right\}} \theta_{j_{1} \ldots j_{k}} \times\right. \\
& \left(\sum _ { l = 0 } ^ { k - 1 } \sum _ { ( a 1 , \ldots , a _ { k } ) _ { - i } \in A _ { l , k - 1 } } \left\{(-1)^{l+1} 2^{k-1-l} \bar{H}_{i}\left(\operatorname{VaR}_{\kappa}\left(S_{n}\right) ; 2^{a_{1}} \lambda_{j_{1}} ; \ldots ; 2^{a_{k}} \lambda_{j_{k}} ; \lambda_{i_{k+1}} ; \ldots \lambda_{i_{n}}\right)\right.\right. \\
& \left.\left.+(-1)^{l} 2^{k-1-l} \bar{G}_{i}\left(\operatorname{VaR} R_{\kappa}\left(S_{n}\right) ; 2^{a_{1}} \lambda_{j_{1}} ; \ldots ; 2^{a_{k}} \lambda_{j_{k}} ; \lambda_{i_{k+1}} ; \ldots \lambda_{i_{n}}\right)\right\}\right) \\
& +\sum_{k=2}^{n-1} \sum_{1 \leq j_{1}<\ldots<j_{k} \leq n \bigcap i \notin\left\{j_{1}, \ldots, j_{k}\right\}} \theta_{j_{1} \ldots j_{k}} \times \\
& \left.\left(\sum_{l=0}^{k} \sum_{\left(a 1, \ldots, a_{k}\right) \in A_{l, k}}(-1)^{l} 2^{k-l} \bar{H}_{i}\left(\operatorname{VaR}_{\kappa}\left(S_{n}\right) ; 2^{a_{1}} \lambda_{j_{1}} ; \ldots ; 2^{a_{k}} \lambda_{j_{k}} ; \lambda_{i_{k+1}} ; \ldots \lambda_{i_{n}}\right)\right)\right],
\end{aligned}
$$

where $\bar{H}_{i}\left(x ; \gamma_{1}, \ldots, \gamma_{i-1}, \gamma_{i+1}, \ldots, \gamma_{n}\right)$ and $\bar{G}_{i}\left(x ; \gamma_{1}, \ldots, \gamma_{i-1}, \gamma_{i+1}, \ldots, \gamma_{n}\right)$ are the $(n-2)$-th order divided differences of respectively $\bar{H}_{i}(x ; \gamma)=\frac{x e^{-\gamma_{i} x}}{\gamma_{i}\left(\gamma_{i}-\gamma\right)}+\frac{e^{-\gamma_{i} x}}{\gamma_{i}^{2}\left(\gamma_{i}-\gamma\right)}+\frac{\gamma e^{-\gamma_{i} x}-\gamma_{i} e^{-\gamma x}}{\gamma \gamma_{i}\left(\gamma_{i}-\gamma\right)^{2}}$ and $\bar{G}_{i}(x ; \gamma)=$ $2\left(\frac{x e^{-2 \gamma_{i} x}}{2 \gamma_{i}\left(2 \gamma_{i}-\gamma\right)}+\frac{e^{-2 \gamma_{i} x}}{\left(2 \gamma_{i}\right)^{2}\left(2 \gamma_{i}-\gamma\right)}+\frac{\gamma e^{-2 \gamma_{i} x}-2 \gamma_{i} e^{-\gamma x}}{\gamma 2 \gamma_{i}\left(2 \gamma_{i}-\gamma\right)^{2}}\right), \Lambda=\lambda_{1} \times \ldots \times \lambda_{n}$ is the product of the parameters of exponential distributions, $i_{k+1}, \ldots, i_{n}$ are the missing indexes of $j_{1}, \ldots, j_{k}$ to complete $1, \ldots, n$ and $A_{l, k}$ are the sets of $k$-tuples composed of $l$ zeros and $(k-l)$ ones, for $l=0,1, \ldots, k$ and $k=2, \ldots, n$.

Proof. The capital attributed to the continuous distributed risk $i$ can be expressed as

$$
\begin{aligned}
T \operatorname{TaR} R_{\kappa}\left(X_{i} ; S_{n}\right) & =C T E_{\kappa}\left(X_{i} \mid S_{n}\right) \\
& =\frac{1}{\bar{F}_{S_{n}}\left(\operatorname{VaR}_{\kappa}\left(S_{n}\right)\right)} \int_{\operatorname{VaR}_{\kappa}\left(S_{n}\right)}^{\infty} \int_{0}^{s} x_{i} f_{X_{i}, S_{n}}\left(x_{i}, s\right) d x d s
\end{aligned}
$$

A recursive formula for $f_{X_{i}, S_{n}}\left(x_{i}, s\right)=f_{X_{i}, S_{n}-X_{i}}\left(x_{i}, s-x_{i}\right)$ is needed to evaluate this expression. Given that the risk random variables here are not independent, we cannot directly separate $f_{X_{i}, S_{n}-X_{i}}\left(x_{i}, s-x_{i}\right)$ into the product of $f_{X_{i}}\left(x_{i}\right)$ and $f_{S_{n-1}}\left(s-x_{i}\right)$.

First, we have

$$
\begin{aligned}
f_{X_{i}, S_{n}-X_{i}}\left(x_{i}, s-x_{i}\right)= & f_{X_{i}, X_{1}+X_{2}+\ldots+X_{i-1}+X_{i+1}+\ldots+X_{n}}\left(x_{i}, s-x_{i}\right) \\
= & \int_{0}^{s-x_{i}} \int_{0}^{s-x_{i}-x_{1}} \int_{0}^{s-x_{i}-x_{1}-x_{2}} \ldots \int_{0}^{s-x_{1}-\ldots-x_{n-1}} f_{X_{1}, X_{2}, \ldots, X_{n-1}, X_{n}}\left(x_{1}, x_{2}, \ldots, x_{n-1}, s-x_{1}-\ldots-x_{n-1}\right) \\
& \times d x_{1} d x_{2} \ldots d x_{i-1} d x_{i+1} \ldots d x_{n-1}
\end{aligned}
$$


with $f_{X_{1}, X_{2}, \ldots, X_{n}}\left(x_{1}, x_{2}, \ldots, x_{n}\right)$ defined as in (9) and which can be extended to

$$
\begin{aligned}
& f_{X_{1}, X_{2}, \ldots, X_{n}}\left(x_{1}, x_{2}, \ldots, x_{n}\right)=f_{X_{i}}\left(x_{i}\right)\left(\nu_{-i}\left(x_{1} ; x_{2} ; \ldots ; x_{n} ; \lambda_{1} ; \lambda_{2} ; \ldots ; \lambda_{n}\right)\right. \\
& \quad+\sum_{k=2}^{n} \sum_{1 \leq j_{1}<\ldots<j_{k} \leq n \bigcap \in\left\{j_{1}, \ldots, j_{k}\right\}} \theta_{j_{1} j_{2} \ldots j_{k}}\left(1-2 F_{X_{i}}\left(x_{i}\right)\right) \\
& \quad \times\left[\sum_{l=0}^{k} \sum_{\left(a_{1}, \ldots, a_{k}\right)_{-i} \in A_{l, k-1}}(-1)^{l} \nu_{-i}\left(x_{j_{1}} ; x_{j_{2}} ; \ldots ; x_{j_{k}} ; x_{i_{k+1}} ; \ldots ; x_{i_{n}} ; 2^{a_{1}} \lambda_{j_{1}} ; 2^{a_{2}} \lambda_{j_{2}} ; \ldots ; 2^{a_{k}} \lambda_{j_{k}} ; \lambda_{i_{k+1}} ; \ldots ; \lambda_{i_{n}}\right)\right] \\
& \quad+\sum_{k=2}^{n} \sum_{1 \leq j_{1}<\ldots<j_{k} \leq n \bigcap i \notin\left\{j_{1}, \ldots, j_{k}\right\}} \theta_{j_{1} j_{2} \ldots j_{k}} \\
& \quad \times\left[\sum_{l=0}^{k} \sum_{\left(a_{1}, \ldots, a_{k}\right)_{-i} \in A_{l, k-1}}(-1)^{l} \nu_{-i}\left(x_{j_{1}}, x_{j_{2}} ; \ldots ; x_{j_{k}} ; x_{i_{k+1}} ; \ldots ; x_{i_{n}} ; 2^{a_{1}} \lambda_{j_{1}} ; 2^{a_{2}} \lambda_{j_{2}} ; \ldots ; 2^{a_{k}} \lambda_{j_{k}} ; \lambda_{i_{k+1}} ; \ldots ; \lambda_{i_{n}}\right)\right],
\end{aligned}
$$

where $i_{k+1}, \ldots, i_{n}$ are the missing indexes of $j_{1}, \ldots, j_{k}$ to complete $1, \ldots, n, A_{0, k}=\left\{(1,1, . ., 1)_{1 \times k}\right\}$, $A_{1, k}=\left\{(1,1, . ., 0)_{1 \times k}, \ldots,(0,1, . ., 1)_{1 \times k}\right\}, A_{2, k}=\left\{(1,1, . ., 0,0)_{1 \times k}, \ldots,(0,0, . ., 1)_{1 \times k}\right\}, \ldots, A_{k, k}=$ $\left\{(0,0, . ., 0)_{1 \times k}\right\}$ and

$\nu_{-i}\left(x_{1} ; x_{2} ; \ldots ; x_{n} ; \gamma_{1} ; \gamma_{2} ; \ldots ; \gamma_{n}\right)=\gamma_{1} e^{-\gamma_{1} x_{1}} \times \gamma_{2} e^{-\gamma_{2} x_{2}} \times \ldots \times \gamma_{i-1} e^{-\gamma_{i-1} x_{i-1}} \times \gamma_{i+1} e^{-\gamma_{i+1} x_{i+1}} \times \ldots \times \gamma_{n} e^{-\gamma_{n} x_{n}}$.

As in the proof of the Proposition 4, we use the fact that

$$
\begin{aligned}
& \int_{0}^{s-x_{i}} \int_{0}^{s-x_{i}-x_{1}} \int_{0}^{s-x_{i}-x_{1}-x_{2}} \ldots \int_{0}^{s-x_{1}-\ldots-x_{n-1}} \nu_{-i}\left(x_{1} ; x_{2} ; \ldots ; x_{n} ; \gamma_{1} ; \gamma_{2} ; \ldots ; \gamma_{n}\right) d x_{1} d x_{2} \ldots d x_{i-1} d x_{i+1} \ldots d x_{n-1} \\
& =\sum_{\substack{j=1 \\
j \neq i}}^{n}\left(\prod_{\substack{k=1, k \neq j \\
k \neq i}}^{n} \frac{\gamma_{k}}{\gamma_{k}-\gamma_{j}}\right) \gamma_{j} e^{-\gamma_{j}\left(s-x_{i}\right)} \\
& =h_{-i}\left(s-x_{i} ; \gamma_{1}, \gamma_{2}, \ldots \gamma_{n}\right),
\end{aligned}
$$

and we obtain 


$$
\begin{aligned}
& f_{X_{i}, S_{n}-X_{i}}\left(x_{i}, s-x_{i}\right)=f_{X_{i}}\left(x_{i}\right)\left[h_{-i}\left(s-x_{i} ; \lambda_{1}, \ldots, \lambda_{i-1}, \lambda_{i+1}, \ldots, \lambda_{n}\right)\right. \\
& +\sum_{k=2}^{n} \sum_{1 \leq j_{1}<\ldots<j_{k} \leq n \bigcap \in\left\{j_{1}, \ldots, j_{k}\right\}} \theta_{j_{1} j_{2} \ldots j_{k}}\left(1-2 F_{X_{i}}(x)\right) \\
& \times\left(\sum_{l=0}^{k} \sum_{\left(a_{1}, \ldots, a_{k}\right)_{-i} \in A_{l, k-1}}(-1)^{l} h_{-i}\left(s-x_{i} ; 2^{a_{1}} \lambda_{j_{1}}, \ldots, 2^{a_{k}} \lambda_{j_{k}}, \lambda_{i_{k+1}}, \ldots \lambda_{i_{n}}\right)\right) \\
& +\sum_{k=2}^{n} \sum_{1 \leq j_{1}<\ldots<j_{k} \leq n \bigcap i \notin\left\{j_{1}, \ldots, j_{k}\right\}} \theta_{j_{1} j_{2} \ldots j_{k}} \\
& \left.\times\left(\sum_{l=0}^{k} \sum_{\left(a_{1}, \ldots, a_{k}\right) \in A_{l, k}}(-1)^{l} h_{-i}\left(s-x_{i} ; 2^{a_{1}} \lambda_{j_{1}}, \ldots, 2^{a_{k}} \lambda_{j_{k}}, \lambda_{i_{k+1}}, \ldots \lambda_{i_{n}}\right)\right)\right] .
\end{aligned}
$$

Using the divided difference as in Chiragiev and Landsman (2007), notice that $h_{-i}\left(x ; \gamma_{1}, \ldots, \gamma_{i-1}, \gamma_{i+1}, \ldots, \gamma_{n}\right)=(-1)^{n-2} \times \gamma_{1} \times \ldots \times \gamma_{i-1} \times \gamma_{i+1} \times \ldots \gamma_{n} \times \bar{F}\left(x ; \gamma_{1} ; \ldots ; \gamma_{i-1} ; \gamma_{i+1} ; \ldots ; \gamma_{n}\right)$, where $\bar{F}\left(x ; \gamma_{1} ; \ldots ; \gamma_{i-1} ; \gamma_{i+1} ; \ldots ; \gamma_{n}\right)$ is the $(n-2)$-th order divided difference of $\bar{F}(x ; \gamma)=e^{-\gamma x}$.

Then, we have

$$
\begin{aligned}
\int_{0}^{s} x_{i} f_{X_{i}}\left(x_{i}\right) h_{-i}\left(s-x_{i} ; \lambda_{1}, \ldots, \lambda_{i-1}, \lambda_{i+1}, \ldots, \lambda_{n}\right) d x_{i} & =\int_{0}^{s} x_{i} f_{X_{i}}\left(x_{i}\right)(-1)^{n-2} \Lambda_{-i} \bar{F}\left(x_{i} ; \lambda_{1} ; \ldots ; \lambda_{i-1} ; \lambda_{i+1} ; \ldots ; \lambda_{n}\right) d x_{i} \\
& =(-1)^{n-1} \Lambda H_{i}\left(s ; \lambda_{1} ; \ldots ; \lambda_{i-1} ; \lambda_{i+1} ; \ldots ; \lambda_{n}\right)
\end{aligned}
$$

where $\Lambda_{-i}=\lambda_{1} \times \ldots \times \lambda_{i-1} \times \lambda_{i+1} \times \ldots \times \lambda_{n}, \Lambda=\lambda_{1} \times \lambda_{2} \times \ldots \times \lambda_{n}$ and

$$
H_{i}(s ; \lambda)=-\int_{0}^{s} x_{i} e^{-\lambda_{i} x_{i}} \bar{F}\left(s-x_{i} ; \lambda\right) d x_{i} .
$$

Let also

$$
G_{i}(s ; \lambda)=-\int_{0}^{s} 2 x_{i} e^{-2 \lambda_{i} x_{i}} \bar{F}\left(s-x_{i} ; \lambda\right) d x_{i} .
$$

Given that the risks here are exponentially distributed, $H_{i}$ and $G_{i}$ take the form

$$
\begin{aligned}
H_{i}(s ; \lambda) & =-\int_{0}^{s} x_{i} e^{-\lambda_{i} x_{i}} \bar{F}\left(s-x_{i} ; \lambda\right) d x_{i} \\
& =-e^{-\lambda s} \int_{0}^{s} x_{i} e^{-\left(\lambda_{i}-\lambda\right) x_{i}} d s \\
& =\frac{s e^{-\lambda_{i} s}}{\lambda_{i}-\lambda}+\frac{e^{-\lambda_{i} s}}{\left(\lambda_{i}-\lambda\right)^{2}}-\frac{e^{-\lambda s}}{\left(\lambda_{i}-\lambda\right)^{2}}
\end{aligned}
$$


and

$$
\begin{aligned}
G_{i}(s ; \lambda) & =-\int_{0}^{s} 2 x_{i} e^{-2 \lambda_{i} x_{i}} \bar{F}\left(s-x_{i} ; \lambda\right) d x_{i} \\
& =-2 e^{-\lambda s} \int_{0}^{s} x_{i} e^{-\left(2 \lambda_{i}-\lambda\right) x_{i}} d s \\
& =2\left(\frac{s e^{-2 \lambda_{i} s}}{2 \lambda_{i}-\lambda}+\frac{e^{-2 \lambda_{i} s}}{\left(2 \lambda_{i}-\lambda\right)^{2}}-\frac{e^{-\lambda s}}{\left(2 \lambda_{i}-\lambda\right)^{2}}\right) .
\end{aligned}
$$

Using (13), we can express $\int_{0}^{s} x_{i} f_{X_{i}, S_{n}}\left(x_{i}, s\right) d x_{i}$ as

$$
\begin{aligned}
& \int_{0}^{s} x f_{X_{i}, S_{n}}(x, s) d x=(-1)^{n-1} \Lambda\left[H_{i}\left(s ; \lambda_{1} ; \ldots ; \lambda_{n}\right)+\sum_{k=2}^{n} \sum_{1 \leq j_{1}<\ldots<j_{k} \leq n \bigcap i \in\left\{j_{1}, \ldots, j_{k}\right\}} \theta_{j_{1} \ldots j_{k}} \times\right. \\
& \left\{\sum _ { l = 0 } ^ { k - 1 } \sum _ { ( a 1 , \ldots , a _ { k } ) _ { - i } \in A _ { l , k - 1 } } \left((-1)^{l+1} 2^{k-1-l} H_{i}\left(s ; 2^{a_{1}} \lambda_{j_{1}} ; \ldots ; 2^{a_{k}} \lambda_{j_{k}} ; \lambda_{i_{k+1}} ; \ldots \lambda_{i_{n}}\right)\right.\right. \\
& \left.\left.+(-1)^{l} 2^{k-1-l} G_{i}\left(s ; 2^{a_{1}} \lambda_{j_{1}} ; \ldots ; 2^{a_{k}} \lambda_{j_{k}} ; \lambda_{i_{k+1}} ; \ldots \lambda_{i_{n}}\right)\right)\right\} \\
& +\sum_{k=2}^{n-1} \sum_{1 \leq j_{1}<\ldots<j_{k} \leq n \bigcap i \notin\left\{j_{1}, \ldots, j_{k}\right\}} \theta_{j_{1} \ldots j_{k}} \times \\
& \left.\left\{\sum_{l=0}^{k} \sum_{\left(a 1, \ldots, a_{k}\right) \in A_{l, k}}(-1)^{l} 2^{k-l} H_{i}\left(s ; 2^{a_{1}} \lambda_{j_{1}} ; \ldots ; 2^{a_{k}} \lambda_{j_{k}} ; \lambda_{i_{k+1}} ; \ldots \lambda_{i_{n}}\right)\right\}\right] .
\end{aligned}
$$

To calculate the risk contribution as in (11), the $H_{i}(s)$ and $G_{i}(s)$ terms in (14) must be integrated on $s$ as follows

$$
\begin{aligned}
\bar{H}_{i}(V ; \lambda) & =\int_{V}^{\infty} H_{i}(s ; \lambda) d s \\
& =\int_{V}^{\infty}\left(\frac{s e^{-\lambda_{i} s}}{\lambda_{i}-\lambda}+\frac{e^{-\lambda_{i} s}}{\left(\lambda_{i}-\lambda\right)^{2}}-\frac{e^{-\lambda s}}{\left(\lambda_{i}-\lambda\right)^{2}}\right) d s \\
& =\frac{V e^{-\lambda_{i} V}}{\lambda_{i}\left(\lambda_{i}-\lambda\right)}+\frac{e^{-\lambda_{i} V}}{\lambda_{i}^{2}\left(\lambda_{i}-\lambda\right)}+\frac{\lambda e^{-\lambda_{i} V}-\lambda_{i} e^{-\lambda V}}{\lambda \lambda_{i}\left(\lambda_{i}-\lambda\right)^{2}}
\end{aligned}
$$

and

$$
\begin{aligned}
\bar{G}_{i}(V ; \lambda) & =\int_{V}^{\infty} G_{i}(s ; \lambda) d s \\
& =\int_{V}^{\infty} 2\left(\frac{s e^{-2 \lambda_{i} s}}{2 \lambda_{i}-\lambda}+\frac{e^{-2 \lambda_{i} s}}{\left(2 \lambda_{i}-\lambda\right)^{2}}-\frac{e^{-\lambda s}}{\left(2 \lambda_{i}-\lambda\right)^{2}}\right) d s \\
& =2\left(\frac{V e^{-2 \lambda_{i} V}}{2 \lambda_{i}\left(2 \lambda_{i}-\lambda\right)}+\frac{e^{-2 \lambda_{i} V}}{\left(2 \lambda_{i}\right)^{2}\left(2 \lambda_{i}-\lambda\right)}+\frac{\lambda e^{-2 \lambda_{i} V}-2 \lambda_{i} e^{-\lambda V}}{\lambda 2 \lambda_{i}\left(2 \lambda_{i}-\lambda\right)^{2}}\right) .
\end{aligned}
$$


Finally, expression (11) for $T V a R_{\kappa}\left(X_{i} ; S_{n}\right)$ is obtained

$$
\begin{aligned}
\operatorname{TVaR}_{\kappa}\left(X_{i} ; S_{n}\right)= & \frac{(-1)^{n-1} \Lambda}{1-\kappa}\left[\bar{H}_{i}\left(\operatorname{VaR}_{\kappa}\left(S_{n}\right) ; \lambda_{1} ; \ldots ; \lambda_{n}\right)+\sum_{k=2}^{n} \sum_{1 \leq j_{1}<\ldots<j_{k} \leq n \bigcap i \in\left\{j_{1}, \ldots, j_{k}\right\}} \theta_{j_{1} \ldots j_{k}} \times\right. \\
& \left(\sum _ { l = 0 } ^ { k - 1 } \sum _ { ( a 1 , \ldots , a _ { k } ) _ { - i } \in A _ { l , k - 1 } } \left\{(-1)^{l+1} 2^{k-1-l} \bar{H}_{i}\left(\operatorname{VaR}_{\kappa}\left(S_{n}\right) ; 2^{a_{1}} \lambda_{j_{1}} ; \ldots ; 2^{a_{k}} \lambda_{j_{k}} ; \lambda_{i_{k+1}} ; \ldots \lambda_{i_{n}}\right)\right.\right. \\
& \left.\left.+(-1)^{l} 2^{k-1-l} \bar{G}_{i}\left(\operatorname{VaR} R_{\kappa}\left(S_{n}\right) ; 2^{a_{1}} \lambda_{j_{1}} ; \ldots ; 2^{a_{k}} \lambda_{j_{k}} ; \lambda_{i_{k+1}} ; \ldots \lambda_{i_{n}}\right)\right\}\right) \\
& +\sum_{k=2}^{n-1} \sum_{1 \leq j_{1}<\ldots<j_{k} \leq n \bigcap i \notin\left\{j_{1}, \ldots, j_{k}\right\}} \theta_{j_{1} \ldots j_{k}} \times \\
& \left.\left(\sum_{l=0}^{k} \sum_{\left(a 1, \ldots, a_{k}\right) \in A_{l, k}}(-1)^{l} 2^{k-l} \bar{H}_{i}\left(\operatorname{VaR} R_{\kappa}\left(S_{n}\right) ; 2^{a_{1}} \lambda_{j_{1}} ; \ldots ; 2^{a_{k}} \lambda_{j_{k}} ; \lambda_{i_{k+1}} ; \ldots \lambda_{i_{n}}\right)\right)\right] .
\end{aligned}
$$

Remark 6 To obtain the TVaR-based contribution (6) in the bivariate case from (10), we just have to use the equalities

$$
\begin{aligned}
& \overline{H_{1}}\left(x ; \lambda_{2}\right)=-\frac{1}{\lambda_{1} \lambda_{2}} \xi\left(x ; \lambda_{1} ; \lambda_{2}\right), \\
& \overline{H_{1}}\left(x ; 2 \lambda_{2}\right)=-\frac{1}{\lambda_{1} 2 \lambda_{2}} \xi\left(x ; \lambda_{1} ; 2 \lambda_{2}\right), \\
& \overline{G_{1}}\left(x ; \lambda_{2}\right)=-2 \frac{1}{2 \lambda_{1} \lambda_{2}} \xi\left(x ; 2 \lambda_{1} ; \lambda_{2}\right), \\
& \overline{G_{1}}\left(x ; 2 \lambda_{2}\right)=-2 \frac{1}{2 \lambda_{1} 2 \lambda_{2}} \xi\left(x ; 2 \lambda_{1} ; 2 \lambda_{2}\right) .
\end{aligned}
$$

\subsection{Numerical application}

We illustrate here our results with a numerical example for the bivariate exponential case. Suppose that the parameters of the distributions of $X_{1}$ and $X_{2}$ are respectively $\lambda_{1}=1 / 2$ and $\lambda_{2}=1 / 3$. Let us calculate the VaR, TVaR and TVaR-based allocations for $X_{1}$ and $X_{2}$ for different risk levels $\kappa$ and different FGM copula parameters $\theta$. We write bellow the cumulative distribution function of $S$ which can be expressed in the current case as a combination of generalized Erlang cdf's

$$
F_{S}(s)=(1+\theta) H\left(s ; \lambda_{1} ; \lambda_{2}\right)-\theta H\left(s ; 2 \lambda_{1} ; \lambda_{2}\right)-\theta H\left(s ; \lambda_{1} ; 2 \lambda_{2}\right)+\theta H\left(s ; 2 \lambda_{1} ; 2 \lambda_{2}\right),
$$

where $H\left(s ; \lambda_{1} ; \lambda_{2}\right)=\frac{\lambda_{2}}{\lambda_{2}-\lambda_{1}}\left(1-e^{-\lambda_{1} s}\right)+\frac{\lambda_{1}}{\lambda_{1}-\lambda_{2}}\left(1-e^{-\lambda_{2} s}\right)$ is the cdf of a 2-generalized Erlang distribution with parameters $\left(\lambda_{1}, \lambda_{2}\right)$. The numerical results for the VaR, TVaR and TVaR-based allocations are displayed in Tables 1,2 and 3. 


\begin{tabular}{|c|c|c|c|c|c|}
\hline$\theta=-1$ & $\kappa=0.5$ & $\kappa=0.75$ & $\kappa=0.95$ & $\kappa=0.99$ & $\kappa=0.995$ \\
\hline $\operatorname{VaR}_{\kappa}(S)$ & 4.3188 & 6.5053 & 11.0436 & 15.5235 & 17.4860 \\
\hline$T V a R_{\kappa}(S)$ & 7.3270 & 9.3394 & 13.8369 & 18.3810 & 20.3716 \\
\hline$T V a R_{\kappa}\left(X_{1} ; S\right)$ & 2.7244 & 3.1489 & 3.5085 & 3.2649 & 3.0613 \\
\hline$T V a R_{\kappa}\left(X_{2} ; S\right)$ & 4.6026 & 6.1905 & 10.3283 & 15.1161 & 17.3103 \\
\hline
\end{tabular}

Table 1: Bivariate exponential example with $\theta=-1$.

\begin{tabular}{|c|c|c|c|c|c|}
\hline$\theta=0$ & $\kappa=0.5$ & $\kappa=0.75$ & $\kappa=0.95$ & $\kappa=0.99$ & $\kappa=0.995$ \\
\hline $\operatorname{VaR}_{\kappa}(S)$ & 4.1589 & 6.7187 & 11.9994 & 16.9914 & 19.1073 \\
\hline $\operatorname{TVaR}_{\kappa}(S)$ & 7.6589 & 9.9967 & 15.0984 & 20.0310 & 22.1324 \\
\hline$T V a R_{\kappa}\left(X_{1} ; S\right)$ & 2.9206 & 3.5756 & 4.6115 & 5.2234 & 5.4002 \\
\hline TVaR $_{\kappa}\left(X_{2} ; S\right)$ & 4.7383 & 6.4211 & 10.4869 & 14.8075 & 16.7323 \\
\hline
\end{tabular}

Table 2: Bivariate exponential example with $\theta=0$.

\begin{tabular}{|c|c|c|c|c|c|}
\hline$\theta=1$ & $\kappa=0.5$ & $\kappa=0.75$ & $\kappa=0.95$ & $\kappa=0.99$ & $\kappa=0.995$ \\
\hline $\operatorname{VaR}_{\kappa}(S)$ & 3.9328 & 6.9975 & 12.8673 & 18.0635 & 20.2236 \\
\hline $\operatorname{TVaR}_{\kappa}(S)$ & 7.9817 & 10.6369 & 16.0906 & 21.1529 & 23.2818 \\
\hline$T V a R_{\kappa}\left(X_{1} ; S\right)$ & 3.1066 & 3.9947 & 5.4022 & 6.2662 & 6.5272 \\
\hline$T V a R_{\kappa}\left(X_{2} ; S\right)$ & 4.8750 & 6.6422 & 10.6883 & 14.8867 & 16.7546 \\
\hline
\end{tabular}

Table 3: Bivariate exponential example with $\theta=1$. 


\section{TVaR and the TVaR-based allocation with mixtures of expo- nential marginals and the FGM copula}

Let us consider now that we have two risks $X_{1}$ and $X_{2}$ which are distributed as a mixture of exponentials. Their cdf's and pdf's can be written as

$$
\begin{aligned}
& F_{X_{1}}(x)=\alpha_{11}\left(1-e^{-\lambda_{11} x}\right)+\alpha_{12}\left(1-e^{-\lambda_{12} x}\right) \\
& F_{X_{2}}(x)=\alpha_{21}\left(1-e^{-\lambda_{21} x}\right)+\alpha_{22}\left(1-e^{-\lambda_{22} x}\right) \\
& f_{X_{1}}(x)=\alpha_{11} \lambda_{11} e^{-\lambda_{11} x}+\alpha_{12} \lambda_{12} e^{-\lambda_{12} x} \\
& f_{X_{2}}(x)=\alpha_{21} \lambda_{21} e^{-\lambda_{21} x}+\alpha_{22} \lambda_{22} e^{-\lambda_{22} x}
\end{aligned}
$$

where we restrict our model to $\lambda_{1 i} \neq \lambda_{2 j}, \lambda_{1 i} \neq 2 \lambda_{2 j}, \lambda_{11}+\lambda_{12} \neq \lambda_{2 j}, \lambda_{11}+\lambda_{12} \neq 2 \lambda_{2 j}$ and $\lambda_{11}+\lambda_{12} \neq \lambda_{21}+\lambda_{22}$. As for the exponential distribution case, the calculations can be done without these constraints but the results are not presented here. Mixtures of exponential distributions, also called hyper-exponential distributions, belong to the phase-type distribution family, see Neuts (1981) and Asmussen (2000). They can be used to approximate light- or heavy-tailed distributions with completely monotone pdf's and decreasing failure rates as shown in Feldmann and Whitt (1998), Keatinge (1999) and Khayari et al. (2003). The practical form of the mixture of exponential distributions also permits explicit results.

The following two propositions can be proven similarly as Proposition 1 and Proposition 2 in the previous section given that a mixture of exponentials is just an extension of the exponential distribution.

Proposition 7 Let $X_{1}$ and $X_{2}$ be two random variables with mixture of exponential distributions and a joint cdf defined by a bivariate FGM copula as follows

$$
F_{X_{1}, X_{2}}\left(x_{1}, x_{2}\right)=C_{\theta}^{F G M}\left(F_{X_{1}}\left(x_{1}\right), F_{X_{2}}\left(x_{2}\right)\right)
$$

with $\theta \in[-1,1]$. Then, the TVaR of the aggregate risk $S=X_{1}+X_{2}$ at level $\kappa, 0<\kappa<1$, is

$$
\operatorname{TVaR}_{\kappa}(S)=(I+J+K) \times \frac{1}{1-\kappa},
$$

where

$$
\begin{aligned}
I= & \sum_{i=1}^{2} \sum_{j=1}^{2}\left\{\left[\alpha_{1 i} \alpha_{2 j}+\theta\left(\alpha_{1 i} \alpha_{2 j}-2 \alpha_{1 i}^{2} \alpha_{2 j}-2 \alpha_{11} \alpha_{12} \alpha_{2 j}-2 \alpha_{1 i} \alpha_{2 j}^{2}-2 \alpha_{1 i} \alpha_{21} \alpha_{22}\right.\right.\right. \\
& \left.\left.\left.+4 \alpha_{1 i}^{2} \alpha_{2 j}^{2}+4 \alpha_{1 i}^{2} \alpha_{21} \alpha_{22}+4 \alpha_{11} \alpha_{12} \alpha_{2 j}^{2}+4 \alpha_{11} \alpha_{12} \alpha_{21} \alpha_{22}\right)\right] \zeta\left(\operatorname{VaR} R_{\kappa}(S) ; \lambda_{1 i} ; \lambda_{2 j}\right)\right\},
\end{aligned}
$$




$$
\begin{aligned}
J= & \theta \sum_{i=1}^{2} \sum_{j=1}^{2}\left\{\left[\alpha_{1 i}^{2} \alpha_{2 j}-2 \alpha_{1 i}^{2} \alpha_{2 j}^{2}-2 \alpha_{1 i}^{2} \alpha_{21} \alpha_{22}\right] \zeta\left(\operatorname{VaR}_{\kappa}(S) ; 2 \lambda_{1 i} ; \lambda_{2 j}\right)\right. \\
& \left.+\left[2 \alpha_{11} \alpha_{12} \alpha_{2 j}-4 \alpha_{11} \alpha_{12} \alpha_{2 j}^{2}-4 \alpha_{11} \alpha_{12} \alpha_{21} \alpha_{22}\right] \frac{\lambda_{1 i}}{\lambda_{11}+\lambda_{12}} \zeta\left(\operatorname{VaR}_{\kappa}(S) ; \lambda_{11}+\lambda_{12} ; \lambda_{2 j}\right)\right\}
\end{aligned}
$$

and

$$
\begin{aligned}
K= & \theta \sum_{i=1}^{2} \sum_{j=1}^{2}\left\{\left[\alpha_{1 i} \alpha_{2 j}^{2}-2 \alpha_{1 i}^{2} \alpha_{2 j}^{2}-2 \alpha_{11} \alpha_{12} \alpha_{2 j}^{2}\right] \zeta\left(\operatorname{VaR} R_{\kappa}(S) ; \lambda_{1 i} ; 2 \lambda_{2 j}\right)\right. \\
& +\alpha_{1 i}^{2} \alpha_{2 j}^{2} \zeta\left(\operatorname{VaR}_{\kappa}(S) ; 2 \lambda_{1 i} ; 2 \lambda_{2 j}\right) \\
& +\left[2 \alpha_{1 i} \alpha_{21} \alpha_{22}-4 \alpha_{1 i}^{2} \alpha_{21} \alpha_{22}-4 \alpha_{11} \alpha_{12} \alpha_{21} \alpha_{22}\right] \frac{\lambda_{2 j}}{\lambda_{21}+\lambda_{22}} \zeta\left(V a R_{\kappa}(S) ; \lambda_{1 i} ; \lambda_{21}+\lambda_{22}\right) \\
& +2 \alpha_{1 i}^{2} \alpha_{21} \alpha_{22} \frac{\lambda_{2 j}}{\lambda_{21}+\lambda_{22}} \zeta\left(V_{a} R_{\kappa}(S) ; 2 \lambda_{11} ; \lambda_{21}+\lambda_{22}\right)+2 \alpha_{11} \alpha_{12} \alpha_{2 j}^{2} \frac{\lambda_{1 i}}{\lambda_{11}+\lambda_{12}} \zeta\left(V R_{\kappa}(S) ; \lambda_{11}+\lambda_{12} ;+2 \lambda_{2 j}\right) \\
& \left.+4 \alpha_{11} \alpha_{12} \alpha_{21} \alpha_{22} \frac{\lambda_{1 i} \lambda_{2 j}}{\left(\lambda_{11}+\lambda_{12}\right)\left(\lambda_{21}+\lambda_{22}\right)} \zeta\left(\operatorname{VaR}_{\kappa}(S) ; \lambda_{11}+\lambda_{12} ; \lambda_{21}+\lambda_{22}\right)\right\} .
\end{aligned}
$$

Proposition 8 Let $X_{1}$ and $X_{2}$ be two mixture of exponentials distributed random variables with joint cdf defined by a bivariate FGM copula. Then, the TVaR-based contribution of risk $i, i=1,2$, to the aggregate risk $S=X_{1}+X_{2}$ at level $\kappa, 0<\kappa<1$, is

$$
T \operatorname{VaR}_{\kappa}\left(X_{i} ; S\right)=(L+M+N) \times \frac{1}{1-\kappa},
$$

where

$$
\begin{aligned}
L= & \sum_{i=1}^{2} \sum_{j=1}^{2}\left\{\left[\alpha_{1 i} \alpha_{2 j}+\theta\left(\alpha_{1 i} \alpha_{2 j}-2 \alpha_{1 i}^{2} \alpha_{2 j}-2 \alpha_{11} \alpha_{12} \alpha_{2 j}-2 \alpha_{1 i} \alpha_{2 j}^{2}-2 \alpha_{1 i} \alpha_{21} \alpha_{22}\right.\right.\right. \\
& \left.\left.\left.+4 \alpha_{1 i}^{2} \alpha_{2 j}^{2}+4 \alpha_{1 i}^{2} \alpha_{21} \alpha_{22}+4 \alpha_{11} \alpha_{12} \alpha_{2 j}^{2}+4 \alpha_{11} \alpha_{12} \alpha_{21} \alpha_{22}\right)\right] \xi\left(\operatorname{VaR} R_{\kappa}(S) ; \lambda_{1 i} ; \lambda_{2 j}\right)\right\}, \\
M= & \theta \sum_{i=1}^{2} \sum_{j=1}^{2}\left\{\left[\alpha_{1 i}^{2} \alpha_{2 j}-2 \alpha_{1 i}^{2} \alpha_{2 j}^{2}-2 \alpha_{1 i}^{2} \alpha_{21} \alpha_{22}\right] \zeta\left(\operatorname{VaR}_{\kappa}(S) ; 2 \lambda_{1 i} ; \lambda_{2 j}\right)\right. \\
& \left.+\left[2 \alpha_{11} \alpha_{12} \alpha_{2 j}-4 \alpha_{11} \alpha_{12} \alpha_{2 j}^{2}-4 \alpha_{11} \alpha_{12} \alpha_{21} \alpha_{22}\right] \frac{\lambda_{1 i}}{\lambda_{11}+\lambda_{12}} \zeta\left(\operatorname{VaR}_{\kappa}(S) ; \lambda_{11}+\lambda_{12} ; \lambda_{2 j}\right)\right\}
\end{aligned}
$$




$$
\begin{aligned}
N= & \theta \sum_{i=1}^{2} \sum_{j=1}^{2}\left\{\left[\alpha_{1 i} \alpha_{2 j}^{2}-2 \alpha_{1 i}^{2} \alpha_{2 j}^{2}-2 \alpha_{11} \alpha_{12} \alpha_{2 j}^{2}\right] \xi\left(\operatorname{VaR}_{\kappa}(S) ; \lambda_{1 i} ; 2 \lambda_{2 j}\right)\right. \\
& +\alpha_{1 i}^{2} \alpha_{2 j}^{2} \xi\left(\operatorname{VaR}_{\kappa}(S) ; 2 \lambda_{1 i} ; 2 \lambda_{2 j}\right) \\
& +\left[2 \alpha_{1 i} \alpha_{21} \alpha_{22}-4 \alpha_{1 i}^{2} \alpha_{21} \alpha_{22}-4 \alpha_{11} \alpha_{12} \alpha_{21} \alpha_{22}\right] \frac{\lambda_{2 j}}{\lambda_{21}+\lambda_{22}} \xi\left(V_{a} R_{\kappa}(S) ; \lambda_{1 i} ; \lambda_{21}+\lambda_{22}\right) \\
& +2 \alpha_{1 i}^{2} \alpha_{21} \alpha_{22} \frac{\lambda_{2 j}}{\lambda_{21}+\lambda_{22}} \xi\left(s ; 2 \lambda_{1 i} ; \lambda_{21}+\lambda_{22}\right)+2 \alpha_{11} \alpha_{12} \alpha_{2 j}^{2} \frac{\lambda_{1 i}}{\lambda_{11}+\lambda_{12}} \xi\left(\operatorname{VaR} R_{\kappa}(S) ; \lambda_{11}+\lambda_{12} ;+2 \lambda_{2 j}\right) \\
& \left.+4 \alpha_{11} \alpha_{12} \alpha_{21} \alpha_{22} \frac{\lambda_{1 i} \lambda_{2 j}}{\left(\lambda_{11}+\lambda_{12}\right)\left(\lambda_{21}+\lambda_{22}\right)} \xi\left(\operatorname{VaR}_{\kappa}(S) ; \lambda_{11}+\lambda_{12} ; \lambda_{21}+\lambda_{22}\right)\right\} .
\end{aligned}
$$

\section{Approximation methods for TVaR-based allocation}

We have seen in the previous sections that it is possible to have an exact expression for the TVaR of a sum of several dependent random variables and the contribution of each random variable to the aggregate TVaR for some specific situations, in particular when using the FGM copula. For most copulas, it is more complicated to directly calculate this risk measure. Embrechts and Puccetti (2007) proposed an algorithm to compute numerically the cdf of the sum of two random variables joined by a copula. They used an approximation of the set $\left\{\left(x_{1}, x_{2}\right) \in[0,+\infty)^{2}: x_{1}+x_{2} \leq s\right\}$ by a countable union of disjoint rectangles to obtain an evaluation of $F_{S}(s)$ with $S=X_{1}+X_{2}$. In the present paper, we expose a simple alternative to approximate this cumulative distribution function with the use of common discretization methods that can be found in Klugman et al. (2008). Then we evaluate the TVaR and its contributions when the random variables are linked by any copula. The method is here exposed for two random variables but can be expanded to more random variables as shown in the numerical applications.

\subsection{Discretization methods}

We use three discretization methods in our study that are defined just below. For these three methods, we suppose that $X$ is a continuous random variable with cdf $F_{X}$ and that $h$ is the discretization span.

Definition 9 (Lower method) The lower method provides a probability mass function of the discretized random variable $\tilde{X}$ given by

$$
\left\{\begin{array}{l}
f_{\tilde{X}}(0)=0 \\
f_{\tilde{X}}(j h)=F_{X}(j h)-F_{X}((j-1) h), \quad \text { for } j=1,2, \ldots
\end{array}\right.
$$

Definition 10 (Upper method) The upper method provides a probability mass function of the 
discretized random variable $\tilde{X}$ given by

$$
\left\{\begin{array}{l}
f_{\tilde{X}}(0)=F_{X}(h) \\
f_{\tilde{X}}(j h)=F_{X}((j+1) h)-F_{X}(j h), \quad \text { for } j=1,2, \ldots
\end{array}\right.
$$

Definition 11 (Mean preserving method) The mean preserving method provides a probability mass function of the discretized random variable $\tilde{X}$ given by

$$
\left\{\begin{array}{l}
f_{\tilde{X}}(0)=1-\frac{E[X \wedge h]}{h} \\
f_{\tilde{X}}(j h)=\frac{2 E[X \wedge j h]-E[X \wedge(j-1) h]-E[X \wedge(j+1) h]}{h}, \text { for } j=1,2, \ldots .
\end{array}\right.
$$

This method ensures that the mean of the discretized distribution is the same as the original distribution.

Remark 12 It is shown e.g. in Müller and Stoyan (2002) and Denuit et al. (2005) that $X \leq_{s d}$ $\tilde{X}$ under the lower method, $\tilde{X} \leq_{\text {sd }} X$ under the upper method, and $X \leq_{i c x} \tilde{X}$ under the mean preserving method, where $\leq_{s d}$ and $\leq_{i c x}$ designate the stochastic dominance order and the increasing convex order respectively (see the same references for the definitions).

\subsection{The bivariate case}

Suppose that we have two continuous distributed risks $X_{1}$ and $X_{2}$. The joint cdf $F_{X_{1}, X_{2}}$ is defined by a fixed copula $C$ which introduces a dependence structure between the risks. We discretize $X_{1}$ and $X_{2}$ with one of the three methods described before. We denote by $\tilde{X}_{1}, \tilde{X}_{2}$ the discretized random variables obtained and keep the same dependence relation between these two new random variables with the copula $C$. Then, we define $\tilde{S}=\tilde{X}_{1}+\tilde{X}_{2}$ that we use two approximate $S=X_{1}+X_{2}$.

For a constant discretization span $h$, the cdf of $\left(\tilde{X}_{1}, \tilde{X}_{2}\right)$ for $k \geq 0$ and $l \geq 0$ is

$$
F_{\tilde{X}_{1}, \tilde{X}_{2}}(k h, l h)=\sum_{i=0}^{k} \sum_{j=0}^{l} \operatorname{Pr}\left(\tilde{X}_{1}=i h, \tilde{X}_{2}=j h\right) .
$$

The cdf of $\tilde{S}$ for $j \geq 0$ is given by

$$
F_{\tilde{S}}(j h)=\sum_{i=0}^{j} \operatorname{Pr}(\tilde{S}=i h)
$$

where the probability mass function (pmf) of $\tilde{S}$ is

$$
\begin{aligned}
& \operatorname{Pr}(\tilde{S}=0)=\operatorname{Pr}\left(\tilde{X}_{1}=0 ; \tilde{X}_{2}=0\right), \text { and } \\
& \operatorname{Pr}(\tilde{S}=j h)=\sum_{i=0}^{j} \operatorname{Pr}\left(\tilde{X}_{1}=i h ; \tilde{X}_{2}=(j-i) h\right), \text { for } j=1,2, \ldots .
\end{aligned}
$$


The joint pmf of $\left(\tilde{X}_{1}, \tilde{X}_{2}\right)$ is obtained with the copula as follows

$$
\begin{aligned}
\operatorname{Pr}\left(\tilde{X}_{1}=0, \tilde{X}_{2}=0\right)= & C\left(F_{\tilde{X}_{1}}(0), F_{\tilde{X}_{2}}(0)\right), \\
\operatorname{Pr}\left(\tilde{X}_{1}=0, \tilde{X}_{2}=j h\right)= & C\left(F_{\tilde{X}_{1}}(0), F_{\tilde{X}_{2}}(j h)\right)-C\left(F_{\tilde{X}_{1}}(0), F_{\tilde{X}_{2}}((j-1) h)\right), \\
\operatorname{Pr}\left(\tilde{X}_{1}=i h, \tilde{X}_{2}=0\right)= & C\left(F_{\tilde{X}_{1}}(i h), F_{\tilde{X}_{2}}(0)\right)-C\left(F_{\tilde{X}_{1}}((i-1) h), F_{\tilde{X}_{2}}(0)\right), \\
\operatorname{Pr}\left(\tilde{X}_{1}=i h, \tilde{X}_{2}=j h\right)= & C\left(F_{\tilde{X}_{1}}(i h), F_{\tilde{X}_{2}}(j h)\right)-C\left(F_{\tilde{X}_{1}}((i-1) h), F_{\tilde{X}_{2}}(j h)\right) \\
& -C\left(F_{\tilde{X}_{1}}(i h), F_{\tilde{X}_{2}}((j-1) h)\right)+C\left(F_{\tilde{X}_{1}}((i-1) h), F_{\tilde{X}_{2}}((j-1) h)\right) .
\end{aligned}
$$

Then, the TVaR of $S$ can be approximated by the TVaR of $\tilde{S}$ which is given by

$$
\begin{aligned}
T \operatorname{VaR}(\tilde{S}) & =\frac{E\left[\tilde{S} \times 1_{\left\{\tilde{S}>\operatorname{Va} R_{\kappa}(\tilde{S})\right\}}\right]+\operatorname{VaR} R_{\kappa}(\tilde{S})\left(\operatorname{Pr}\left(\tilde{S} \leq \operatorname{VaR} R_{\kappa}(\tilde{S})\right)-\kappa\right)}{1-\kappa} \\
& =\frac{E\left[\tilde{S} \times 1_{\left\{\tilde{S}>k_{0} h\right\}}\right]+k_{0} h\left(\operatorname{Pr}\left(\tilde{S} \leq k_{0} h\right)-\kappa\right)}{1-\kappa},
\end{aligned}
$$

where $\operatorname{VaR}_{\kappa}(\tilde{S})=k_{0} h$.

The TVaR-based allocation of risk $X_{i}$ over the global risk $S$ can be approximated by the TVaRbased allocation of risk $\tilde{X}_{i}$ over the global discretized risk $\tilde{S}$ where

$$
\begin{aligned}
\operatorname{TVaR}_{\kappa}\left(\tilde{X}_{i} ; \tilde{S}\right) & =\frac{E\left[\tilde{X}_{i} \times 1_{\left\{\tilde{S}>V a R_{\kappa}(\tilde{S})\right\}}\right]+\beta_{\tilde{S}} E\left[\tilde{X}_{i} \times 1_{\left\{\tilde{S}=V a R_{\kappa}(\tilde{S})\right\}}\right]}{1-\kappa} \\
& =\frac{E\left[\tilde{X}_{i} \times 1_{\left\{\tilde{S}>k_{0} h\right\}}\right]+\beta_{\tilde{S}} E\left[\tilde{X}_{i} \times 1_{\left\{\tilde{S}=k_{0} h\right\}}\right]}{1-\kappa}, \\
\text { where } \beta_{\tilde{S}}=\left\{\begin{array}{l}
\frac{\operatorname{Pr}\left(\tilde{S} \leq k_{0} h\right)-\kappa}{\operatorname{Pr}\left(\tilde{S}=k_{0} h\right)} \\
0, \text { otherwise. }
\end{array}\right. & \text { if } \operatorname{Pr}\left(\tilde{S}=k_{0} h\right)>0,
\end{aligned}
$$

Remark 13 In corollary 4.6 of Müller and Scarsini (2001), it is shown that if $\left(X_{1}, X_{2}\right)$ and $\left(\widetilde{X}_{1}, \widetilde{X}_{2}\right)$ are random vectors with a common conditionally increasing copula and if $X_{i} \leq_{c x} \widetilde{X}_{i}$ for $i=1,2$, then for all non-negative scalars $a_{1}$ and $a_{2}$ we have

$$
a_{1} X_{1}+a_{2} X_{2} \leq_{c x} a_{1} \widetilde{X}_{1}+a_{2} \widetilde{X}_{2}
$$

This result also holds for the increasing convex order since it is implied by the convex order. From Bäuerle and Müller (2006), it follows that

$$
T \operatorname{VaR} R_{\kappa}\left(a_{1} X_{1}+a_{2} X_{2}\right) \leq T V a R_{\kappa}\left(a_{1} \widetilde{X}_{1}+a_{2} \widetilde{X}_{2}\right)
$$


for $\kappa \in(0,1)$.

\subsection{Numerical applications}

\subsubsection{Bivariate case}

Suppose that $X_{1}$ and $X_{2}$ are exponentially distributed with parameters $\lambda_{1}=1 / 2$ and $\lambda_{2}=1 / 3$ respectively. The dependence between these two risks is defined by the bivariate FGM copula with parameter $\theta^{F G M}=0.8$. This value of $\theta^{F G M}$ implicates a correlation of 0.2 between $X_{1}$ and $X_{2}$. Figure 1 in the appendix illustrates the accuracy of the approximations of the cdf of $S_{2}$ when using the three discretization methods. Indeed, the decrease of the discretization span $h$ implicates a convergence of the discretized cdf to the real one.

The discretization methods allow an approximation of the cdf of $S_{2}$ with any copula. We illustrate the use of the FGM, the Clayton, the Frank and the Gumbel copula. The copulas' parameters are chosen such that we have the same coefficient of correlation between $X_{1}$ and $X_{2}$. Figure 2 in the appendix shows the impact of the copulas on the cdf of $S_{2}$ which is discretized with the mean preserving method when the correlation coefficient is fixed at 0.2 . The first graph displays the drawing of the approximated cdf's of $S_{2}$. We then do the difference between a dependent cdf and the independent cdf and trace this difference for the FGM, the Frank, the Clayton and the Gumbel copula in the second graph of Figure 2. These two graphs highlight the fact that the Clayton copula introduces dependence in lower values and that the Gumbel copula permits dependence in the upper queue.

In Figure 3 in the appendix, we trace the differences between the TVaR of $S_{2}$ using dependent risks with one of the copulas discussed before and the TVaR of $S_{2}$ using the independent copula against the risk level $\kappa$. This is done for three increasing values of correlation between $X_{1}$ and $X_{2}$. Given that the FGM copula only allows weak dependence it just appears on the first graph. The graphs confirm the fact that the Gumbel copula introduces dependence in high values.

Tables 4 and 5 expose the numerical results for the TVaR and the TVaR-based allocation for $X_{1}$ and $X_{2}$ with the four copulas discussed above and confidence levels equal to 0.99 and 0.995 . The calculations are done with the mean preserving discretization method with span $h=0.05$ for the four copulas and also with the exact expression for the FGM copula. The tables attest the good precision of the approximation method and confirm the high dependence values inserted by the Gumbel copula.

\subsubsection{Trivariate case}

We illustrate here the difference between the exact and the approximated methods with three risk variables dependent through a trivariate FGM copula. Suppose that $X_{1}, X_{2}$ and $X_{3}$ are exponentially distributed with parameters $\lambda_{1}=1 / 2, \lambda_{2}=1 / 3$ and $\lambda_{3}=1 / 5$ respectively. The

copula parameters $\theta_{i}^{F G M}, \theta_{i j}^{F G M}$ and $\theta_{i j k}^{F G M}$, for $i=1,2,3, j=1,2,3, k=1,2,3, j \neq i, k \neq i$ 


\begin{tabular}{|l|c|c|c|c|c|}
\hline Copula & $T V a R_{0.99}\left(S_{2}\right)$ & $T V a R_{0.99}\left(X_{1} ; S_{2}\right)$ & $\%$ of $T V a R_{0.99}\left(S_{2}\right)$ & $T V a R_{0.99}\left(X_{2} ; S_{2}\right)$ & $\%$ of $T V a R_{0.99}\left(S_{2}\right)$ \\
\hline FGM exact & 20.9561 & 6.0998 & $29.1 \%$ & 14.8563 & $70.9 \%$ \\
FGM M.P. & 20.9574 & 6.1003 & $29.1 \%$ & 14.8571 & $70.9 \%$ \\
Clayton M.P. & 20.7918 & 5.9419 & $28.6 \%$ & 14.8499 & $71.4 \%$ \\
Frank M.P. & 21.0612 & 6.2158 & $29.5 \%$ & 14.8454 & $70.5 \%$ \\
Gumbel M.P. & 22.9669 & 7.7988 & $34.0 \%$ & 15.1682 & $66 \%$ \\
\hline
\end{tabular}

Table 4: TVaR and TVaR-based allocation for $S_{2}$ with $\kappa=0.99$.

\begin{tabular}{|l|c|c|c|c|c|}
\hline Copula & $T V a R_{0.995}\left(S_{2}\right)$ & $T V a R_{0.995}\left(X_{1} ; S_{2}\right)$ & $\%$ of $T V a R_{0.995}\left(S_{2}\right)$ & $T V a R_{0.995}\left(X_{2} ; S_{2}\right)$ & $\%$ of $T V a R_{0.995}\left(S_{2}\right)$ \\
\hline FGM exact & 23.0839 & 6.3523 & $27.5 \%$ & 16.7316 & $72.5 \%$ \\
FGM M.P. & 23.0859 & 6.3530 & $27.5 \%$ & 16.7329 & $72.5 \%$ \\
Clayton M.P. & 22.9135 & 6.1776 & $27.0 \%$ & 16.7359 & $73.0 \%$ \\
Frank M.P. & 23.2014 & 6.4953 & $28.0 \%$ & 16.7061 & $72.0 \%$ \\
Gumbel M.P. & 26.0088 & 8.9850 & $34.5 \%$ & 17.0237 & $65.5 \%$ \\
\hline
\end{tabular}

Table 5: TVaR and TVaR-based allocation for $S_{2}$ with $\kappa=0.995$.

and $k \neq j$ are all fixed to 1 . As for the bivariate case, we show in Figure 4 of the appendix the convergence to the real cdf of $S_{3}$ of the discretized cdf's when using the three discretization methods.

Tables 6 and 7 compare the numerical results for the TVaR and the TVaR-based allocation for the three risks with confidence levels equal to 0.99 and 0.995 between exact expressions and approximated results using the mean preserving discretization method with span $h=0.3$. As for the bivariate case, they show a satisfying accuracy of the approximation method.

\begin{tabular}{|l|c|c|c|c|}
\hline Method & $T V a R_{0.99}\left(S_{3}\right)$ & $T V a R_{0.99}\left(X_{1} ; S_{3}\right)$ & $T V a R_{0.99}\left(X_{2} ; S_{3}\right)$ & $T V a R_{0.99}\left(X_{3} ; S_{3}\right)$ \\
\hline Exact & 37.5988 & 4.1726 & 8.4033 & 25.0230 \\
Mean P. & 37.6062 & 4.1760 & 8.4060 & 25.0243 \\
\hline
\end{tabular}

Table 6: TVaR and TVaR-based allocation for $S_{3}$ with $\kappa=0.99$.

\section{Conclusion}

This paper introduces the use of copulas in TVaR-based capital allocation. We obtain explicit expressions for the TVaR and TVaR-based allocation for risks that have exponential and mixture of exponentials distributions linked by a FGM copula. The handy form of this copula permits a direct calculation of the coherent risk measure and its decomposition when we suppose only two different risks. In the multivariate situation, we use divided differences as in Chiragiev and Landsman (2007). For other copulas, we present approximations for the TVaR and the TVaR-based allocation using three discretization methods for continuous distributions. 


\begin{tabular}{|l|c|c|c|c|}
\hline Method & $T V a R_{0.995}\left(S_{3}\right)$ & $T V a R_{0.995}\left(X_{1} ; S_{3}\right)$ & $T V a R_{0.995}\left(X_{2} ; S_{3}\right)$ & $T V a R_{0.995}\left(X_{3} ; S_{3}\right)$ \\
\hline Exact & 41.1177 & 4.2044 & 8.6536 & 28.2597 \\
Mean P. & 41.1262 & 4.2080 & 8.6567 & 28.2616 \\
\hline
\end{tabular}

Table 7: TVaR and TVaR-based allocation for $S_{3}$ with $\kappa=0.995$.

\section{Acknowledgements}

The authors acknowledge the Natural Sciences and Engineering Research Council of Canada and the Chaire d'actuariat de l'Universit Laval for their support. This work has also been partially supported by the French Research National Agency (ANR) under the reference ANR-08-BLAN0314-01. The authors would like to thank an anonymous referee for her/his comments. 


\section{APPENDIX}
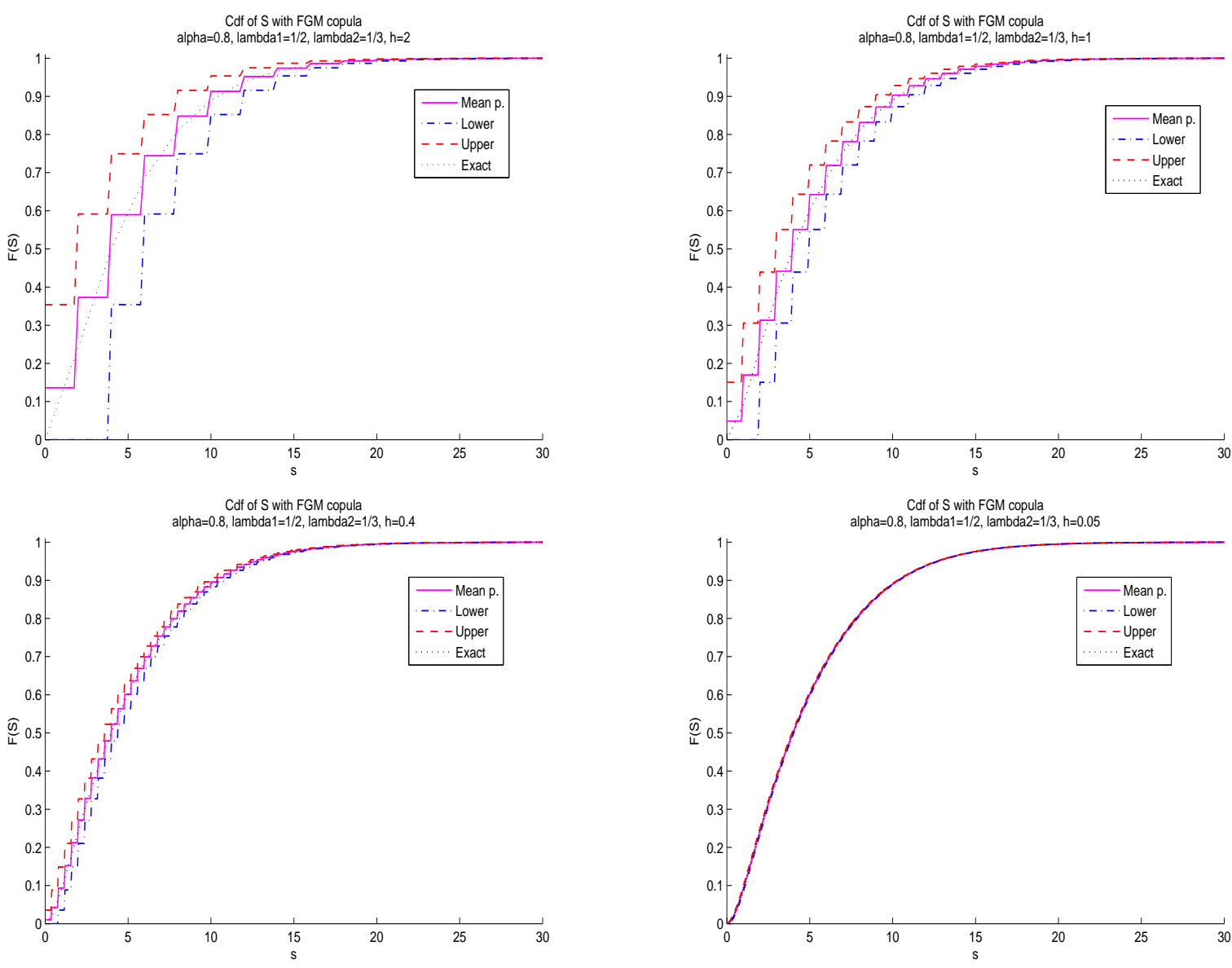

Figure 1: Discretized cdf's vs exact cdf for 2 risks 

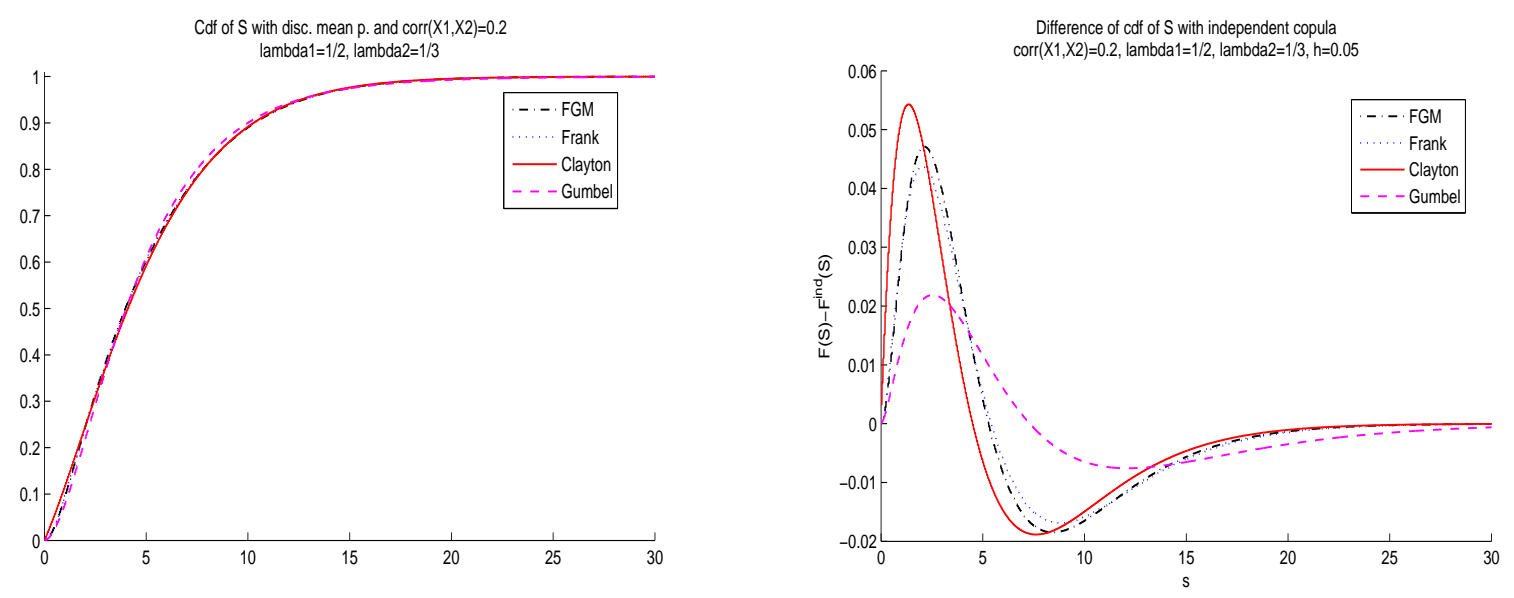

Figure 2: Comparison of copulas with $\operatorname{Corr}\left(X_{1}, X_{2}\right)=0.2$ 

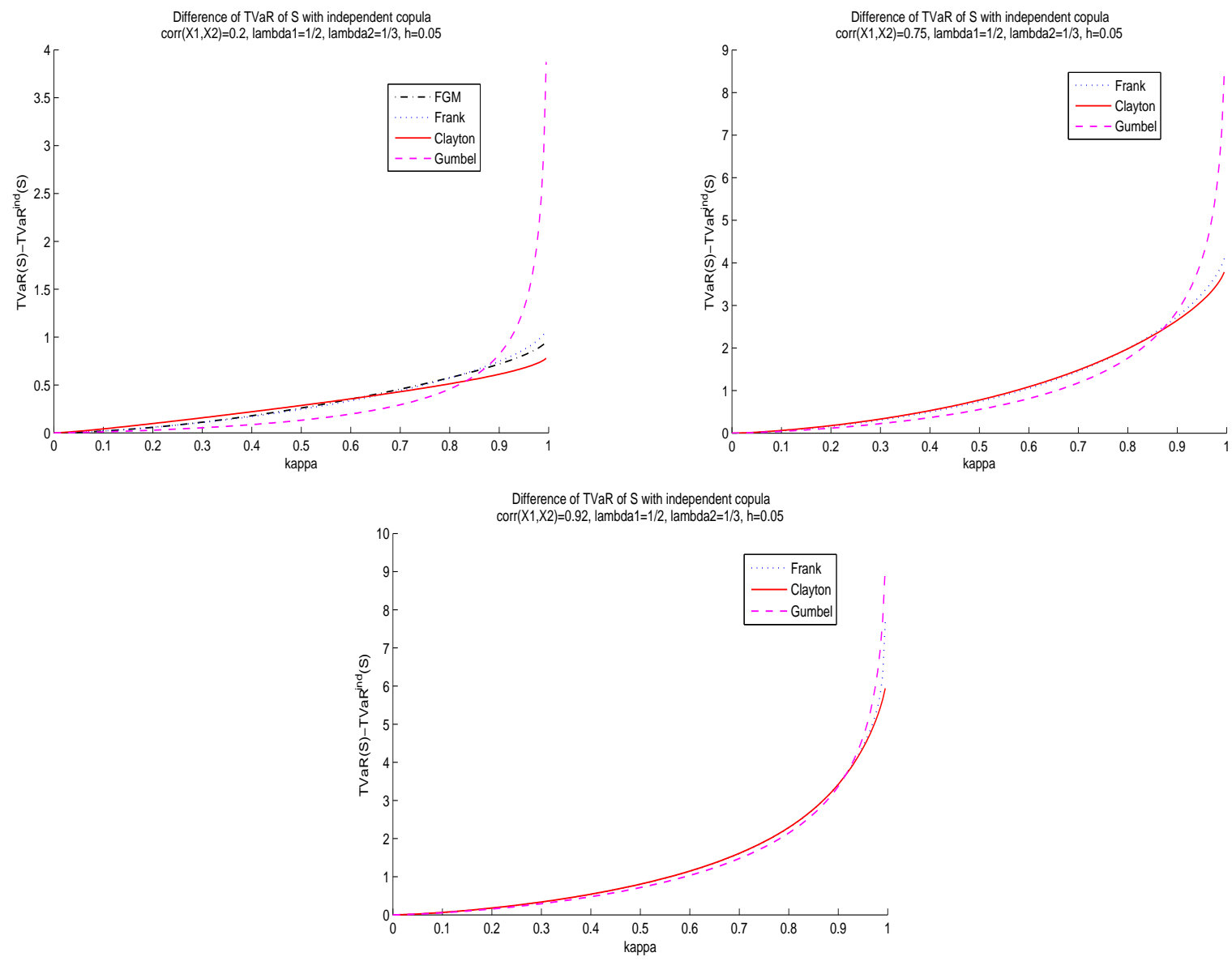

Figure 3: TVaR of S with different copulas and correlation coefficients 

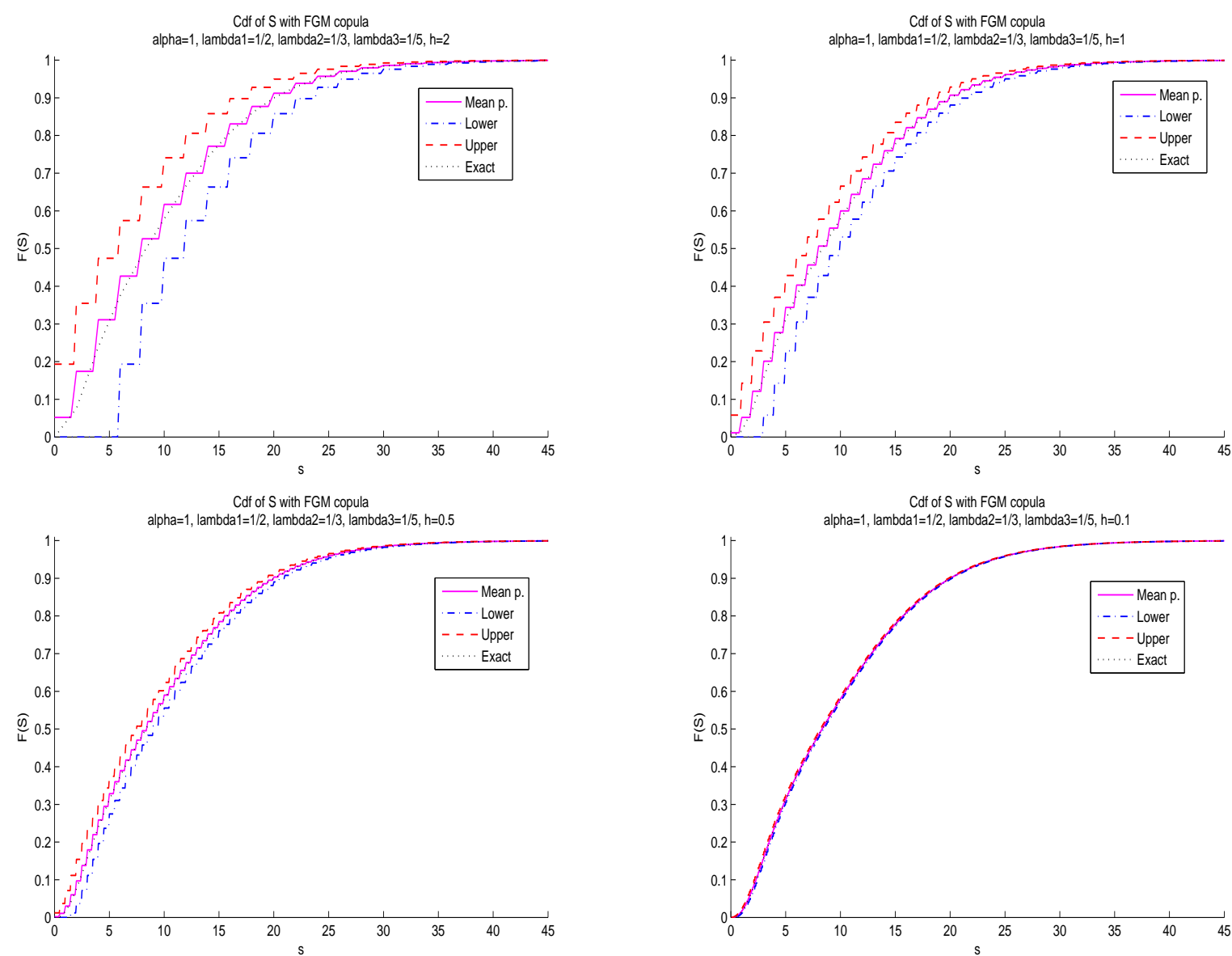

Figure 4: Discretized cdf's vs exact cdf for 3 risks 


\section{References}

Acerbi, C., Nordio, C., and Sirtori, C. (2001). Expected shortfall as a tool for financial risk management. Working paper.

Acerbi, C. and Tasche, D. (2002). On the coherence of expected shortfall. Journal of Banking $E^{6}$ Finance, 26(7):1487-1503.

Artzner, P., Delbaen, F., Eber, J.-M., and Heath, D. (1999). Coherent measures of risk. Math. Finance, 9(3):203-228.

Asmussen, S. (2000). Matrix-analytic models and their analysis. Scand. J. Statist., 27(2):193-226.

Bäuerle, N. and Müller, A. (2006). Stochastic orders and risk measures: consistency and bounds. Insurance Math. Econom., 38(1):132-148.

Cai, J. and Li, H. (2005). Conditional tail expectations for multivariate phase-type distributions. J. Appl. Probab., 42(3):810-825.

CEIOPS (2006). Choice of risk measure for solvency purposes. http://ec.europa.eu/internal_market/insurance/docs/2006-markt-docs/2534-06-annexceiops_en.pdf.

CEIOPS (2007). Draft advice to the European Commission in the framework of the Solvency II Project on pillar I issues - further advice. Consultation paper N20. http://www.ceiops.eu/media/files/consultations/consultationpapers/CP20/CP20.pdf.

Chiragiev, A. and Landsman, Z. (2007). Multivariate pareto portfolios: Tce-based capital allocation and divided differences. Scand. Actuar. J., (4):261-280.

Denuit, M., Dhaene, J., Goovaerts, M. J., and Kaas, R. (2005). Actuarial Theory for Dependent Risks: Measures, Orders and Models. Wiley, New York.

Dhaene, J., Henrard, L., Landsman, Z., Vandendorpe, A., and Vanduffel, S. (2008). Some results on the cte-based capital allocation rule. Insurance Math. Econom., 42(2):855-863.

Embrechts, P. and Puccetti, G. (2007). Fast computation of the distribution of the sum of two dependent random variables. Working paper.

Feldmann, A. and Whitt, W. (1998). Fitting mixtures of exponentials to long-tail distributions to analyze network performance models. Performance Evaluation, 31(3-4):245-279.

Furman, E. and Landsman, Z. (2005). Risk capital decomposition for a multivariate dependent gamma portfolio. Insurance Math. Econom., 37(3):635-649.

Furman, E. and Landsman, Z. (2007). Economic capital allocations for non-negative portfolios of dependent risks. Proceedings of the 37-th International ASTIN Colloquium, Orlando.

Gatfaoui, H. (2005). How does systematic risk impact us credit spreads ? a copula study. Bankers Markets $\&$ Investors, 77:5-12.

Gatfaoui, H. (2007). Credit default swap spreads and u.s. financial market: Investigating some dependence. 20th Australasian Finance \& Banking Conference 2007. 
Keatinge, C. L. (1999). Modeling losses with the mixed exponential distribution. In Proceedings of the Casualty Actuarial Society, volume 86, pages 654-698.

Khayari, R. E. A., Sadre, R., and Haverkort, B. R. (2003). Fitting world-wide web request traces with the em-algorithm. Performance Evaluation, 52(2-3):175 - 191. Internet Performance and Control of Network Systems.

Kim, H. T. (2007). Estimation and allocation of insurance risk capital. PhD thesis, University of Waterloo.

Klugman, S. A., Panjer, H. H., and Willmot, G. E. (2008). Loss models: From data to decisions. Wiley Series in Probability and Statistics. John Wiley \& Sons Inc., Hoboken, NJ, third edition.

Landsman, Z. M. and Valdez, E. A. (2003). Tail conditional expectations for elliptical distributions. N. Am. Actuar. J., 7(4):55-71.

Müller, A. and Scarsini, M. (2001). Stochastic comparison of random vectors with a common copula. Math. Oper. Res., 26(4):723-740.

Müller, A. and Stoyan, D. (2002). Comparison methods for stochastic models and risks. Wiley Series in Probability and Statistics. John Wiley \& Sons Ltd., Chichester.

Nelsen, R. B. (2006). An introduction to copulas. Springer Series in Statistics. Springer, New York, second edition.

Neuts, M. F. (1981). Matrix-geometric solutions in stochastic models, volume 2 of Johns Hopkins Series in the Mathematical Sciences. Johns Hopkins University Press, Baltimore, Md. An algorithmic approach.

Panjer, H. H. (2002). Measurement of risk, solvency requirements and allocation of capital within financial conglomerates. Institute of Insurance and Pension Research, University of Waterloo, Research Report 01-15.

Schmock, U. (2006). Modelling dependent credit risks with extensions of creditrisk+ and application to operational risk. Lecture notes, PRisMa Lab, Institute for Mathematical Methods in Economics, Vienna University of Technology.

Schmock, U. and Straumann, D. (1999). Allocation of risk capital and performance measurement. Talk at the Conference on Quantitative Methods in Finance, Sydney, Australia.

Tasche, D. (1999). Risk contributions and performance measurement. Working paper, Technische Universitt Mnchen.

Wang, S. S. (2002). A set of new methods and tools for enterprise risk capital management and portfolio optimisation. Working paper, SCOR Reinsurance Company.

Yeo, K. L. and Valdez, E. A. (2006). Claim dependence with common effects in credibility models. Insurance Math. Econom., 38(3):609-629. 